\title{
Random polytopes in a convex polytope, independence of shape, and concentration of vertices
}

\author{
Imre Bárány ${ }^{1, \star, \star \star}$ and Christian Buchta ${ }^{2, \star \star \star}$ \\ 1 Cowles Foundation, Yale University, New Haven, CT 06520, USA and Courant Institute of Mathematical \\ Sciences, New York University, NY 10012, USA \\ 2 Institut für Analysis, Technische Mathematik und Versicherungsmathematik, Technische Universität, \\ Wiedner Hauptstrasse 8-10, A-1040 Wien, Austria
}

Received April 10, 1992; in revised form December 22, 1992

Mathematics Subject Classification (1991): 52A22, 60D05

\section{Introduction and main results}

Write $\mathscr{K}^{d}$ for the set of all convex bodies (convex compact sets with nonempty interior) in $\mathbb{R}^{d}$. Define $\mathscr{K}_{1}^{d}$ as the set of those $K \in \mathscr{K}^{d}$ with vol $K=1$. Fix $K \in \mathscr{K}_{1}^{d}$ and choose points $x_{1}, \ldots, x_{n} \in K$ randomly, independently, and according to the uniform distribution on $K$. Then $K_{n}=\operatorname{conv}\left\{x_{1}, \ldots, x_{n}\right\}$ is a random polytope in $K$. Write $E(K, n)$ for the expectation of the random variable $\operatorname{vol}\left(K \backslash K_{n}\right) . E(K, n)$ shows how well $K_{n}$ approximates $K$ in volume on the average.

Groemer [Gr1] proved that, among all convex bodies $K \in \mathscr{K}_{1}^{d}$, the ellipsoids are approximated worst, i.e.

$$
E(K, n) \leq E(B, n)
$$

where $B$ is any ellipsoid of volume one. Equality holds if and only if $K$ is an ellipsoid. Wieacker [Wi] derived that $E(B, n)=\operatorname{const}(d) n^{-2 /(d+1)}+o\left(n^{-2 /(d+1)}\right)$. Affentranger [Af1] developed formulae from which $E(B, n)$ can be computed explicitly.

Here we prove that, among all convex bodies $K \in \mathscr{K}_{1}^{d}$, the simplices are approximated best in the following sense:

Theorem 1. Let $K \in \mathscr{K}_{1}^{d}$ and $\Delta \in \mathscr{K}_{1}^{d}, \Delta$ a simplex, $d \geq 2$. Then

* Mailing address of the first author: Mathematical Institute of the Hungarian Academy of Sciences, P.O. Box 127, H-1364 Budapest, Hurigary.

** Supported by the Program in Discrete Mathematics and Its Applications at Yale and NSF Grant CCR-8901484. Partially supported by the Hungarian National Science Foundation Grant 1812.

$\star \star \star$ Work on this paper was done during a stay at the University of Freiburg im Breisgau supported by Deutscher Akademischer Austauschdienst. 


$$
\lim \inf \frac{E(K, n)}{E(\Delta, n)} \geq 1+\frac{1}{d+1}
$$

unless $K$ is a simplex.

(1.2) shows that for every $K \in \mathscr{K}_{1}^{d}$ different from a simplex there is $n_{0}(K)$ such that for $n \geq n_{0}(K)$

$$
E(K, n) \geq\left(1+\frac{1}{2 d}\right) E(\Delta, n) .
$$

Most probably, for every $K \in \mathscr{K}_{1}^{d}$ and $n \geq d+1$

$$
E(K, n) \geq E(\Delta, n)
$$

with equality if and only if $K$ is a simplex. For $d=2$ and $n=3$ Blaschke [B11], [B12] proved (1.1) with equality if and only if $K$ is an ellipse and (1.3) with equality if and only if $K$ is a triangle, but his remark (not repeated in [B12]) that the method of proof can be extended without difficulty to all dimensions $d$ and $n=d+1$ appears to be erroneous; cf., e.g., Groemer [Gr2], Schneider [Schn], or Pfiefer [Pf]. Blaschke's result was extended to $n=4$ by Buchta [Bu1]. For $d=2$ and $n \geq 3$ Dalla and Larman [DL] proved (1.3) with strict inequality if $K$ is any polygon other than a triangle. Their result was completed by Giannopoulos [Gi] who showed that the inequality is strict whenever $K$ is a plane convex body other than a triangle. The occurring bound was derived by Buchta [Bu2]:

$$
E(\text { triangle }, n)=\frac{2}{n+1} \sum_{k=1}^{n} \frac{1}{k} \text {. }
$$

In higher dimensions, Dalla and Larman [DL] proved (1.3) in the case that $K$ is a $d$-polytope with at most $d+2$ vertices.

Actually, (1.2) separates the simplices from all other convex bodies. This is due to the fact that for polytopes $P \in \mathscr{K}_{1}^{d}$ we can determine $E(P, n)$ up to first order precision. To state this result we call a chain $F_{0} \subset F_{1} \subset \ldots \subset F_{d-1}$ where $F_{i}$ is an $i$-dimensional face of $P(i=0,1, \ldots, d-1)$ a tower of $P$. (Sometimes this is called a (complete) flag; cf., e.g., Bayer and Lee [BaLe].) Write $T(P)$ for the number of towers of $P$.

Theorem 2. Let $P \in \mathscr{K}_{1}^{d}$ be a polytope, $d \geq 2$. Then

$$
E(P, n)=\frac{T(P)}{(d+1)^{d-1}(d-1) !} \frac{\log ^{d-1} n}{n}+O\left(\frac{\log ^{d-2} n \log \log n}{n}\right) .
$$

For a simple polytope $P$, where $T(P)$ is $d$ ! times the number of vertices of $P$, vert $P$, Affentranger and Wieacker [AW] recently proved that

$$
E(P, n)=\frac{d \text { vert } P}{(d+1)^{d-1}} \frac{\log ^{d-1} n}{n}+O\left(\frac{\log ^{d-2} n}{n}\right) .
$$

Before, van Wel [We] deduced for a $d$-dimensional cube and indicated for any simple polytope $P$ that $E(P, n) \sim \operatorname{const}(d)$ vert $P n^{-1} \log ^{d-1} n$ with const $(d)$ expressed by a $\left(d^{2}-d\right)$-fold integral. In the case that $P$ is a tetrahedron $E(P, n) \sim \frac{3}{4} n^{-1} \log ^{2} n$ was 
derived by Buchta [Bu4]. If Efron's identity stated below is taken into consideration, Rényi and Sulanke [RS] much earlier obtained for a polygon $P$ that

$$
E(P, n)=\frac{2}{3} \text { vert } P \frac{\log n}{n}+\frac{\operatorname{const}(P)}{n}+o\left(\frac{1}{n}\right)
$$

with explicitly given const $(P)$.

Estimates for $E(P, n)$ were given in the case that $P$ is a $d$-dimensional cube by Bentley, Kung, Schkolnick and Thompson [BKST] as well as by Devroye [De], in the general case by Dwyer and Kannan [DK], Dwyer [Dw], and Bárány and Larman [BáLa]. The last-mentioned authors proved that $E(P, n)$ is of order $n^{-1} \log ^{d-1} n$ for any polytope $P$.

Denote by $E$ (vert $K_{n}$ ) the expected number of vertices of $K_{n}$. The simple identity due to Efron [Ef]

$$
(n+1) E(K, n)=E\left(\text { vert } K_{n+1}\right) \text { when } K \in \mathscr{K}_{1}^{d}
$$

shows that (1.4) is equivalent to

$$
E\left(\text { vert } P_{n}\right)=\frac{T(P)}{(d+1)^{d-1}(d-1) !} \log ^{d-1} n+O\left(\log ^{d-2} n \log \log n\right) .
$$

The advantage of this formulation is that the assumption vol $K=1$ can be dropped. To prove (1.4), or rather (1.6), we will show that the vertices of $P_{n}$ are "concentrated" in certain simplices associated with towers of $P$. For the precise statement we need some preparation.

Assume that together with the polytope $P \in \mathscr{F}_{1}^{d}$ a hyperplane selection $H(\cdot)$ is given. This is a map that associates with every (nontrivial) face $F$ of $P$ a supporting hyperplane $H(F)$ such that

$$
H(F) \cap P=F \text {. }
$$

Given a tower $T=\left(F_{0}, F_{1}, \ldots, F_{d-1}\right)$ we define the simplex $S(T, \varepsilon)$ associated with $T$ for every small enough $\varepsilon>0$ by induction on $d$. For $d=1$, when $P=[0,1]$, say, and $H(\cdot)$ is unique, we set

$$
\begin{aligned}
& S(0, \varepsilon)=[0, \varepsilon], \\
& S(1, \varepsilon)=[1-\varepsilon, 1] .
\end{aligned}
$$

Assume $S$ has been defined for polytopes $Q \in \mathscr{K}_{1}^{d-1}$. Let $P \in \mathscr{K}_{1}^{d}, T=$ $\left(F_{0}, \ldots, F_{d-1}\right)$ a tower of $P$. For notational convenience we assume that $F_{0}=\{0\}$. Write cone $P$ for the minimal (convex) cone containing $P$ (with apex at the origin). Set $H_{i}=H\left(F_{i}\right)$, and consider the hyperplane $H_{0}(t)$ parallel to $H_{0}$ at a distance $t$ and on the same side of $H_{0}$ as $P$. Then

$$
Q(t):=\text { cone } P \cap H_{0}(t)
$$

is a $(d-1)$-dimensional polytope. Since $\operatorname{vol}_{d-1} Q(t)=\operatorname{const}(P) t^{d-1}$, there is a unique $t_{0}>0$ with $\operatorname{vol}_{a-1} Q\left(t_{0}\right)=1$. Define

$$
Q:=Q\left(t_{0}\right) \in \mathscr{K}_{1}^{d-1}
$$

For a face $F$ of $P$ with $0 \in F$ but $F \neq\{0\}$ the set cone $F \cap H_{0}\left(t_{0}\right)$ is a face of $Q$. Moreover, all faces of $Q$ are of this form. Correspondingly, the tower $T=T_{P}$ gives rise to a tower $T_{Q}$ of $Q$ via 


$$
T_{Q}=\left(\text { cone } F_{1} \cap H_{0}\left(t_{0}\right), \text { cone } F_{2} \cap H_{0}\left(t_{0}\right), \ldots, \text { cone } F_{d-1} \cap H_{0}\left(t_{0}\right)\right) \text {, }
$$

and $H_{P}(\cdot)$ gives rise to a hyperplane selection $H_{Q}(\cdot)$ via

$$
H_{Q}\left(\text { cone } F \cap H_{0}\left(t_{0}\right)\right)=H_{P}(F) \cap H_{0}\left(t_{0}\right)
$$

where $F$ is a face of $P$ with $0 \in F, F \neq\{0\}$. Then, by the induction hypothesis, the simplex $S_{Q}\left(T_{Q}, \varepsilon\right)$ has been defined. Set

$$
S_{P}\left(T_{P}, \varepsilon\right)=\text { cone } S_{Q}\left(T_{Q}, \varepsilon\right) \cap H_{0}(0, \varepsilon)
$$

where $H_{0}(0, t)$ denotes the slab between the hyperplanes $H_{0}$ and $H_{0}(t)$.

Although $S(T, \varepsilon)$ seems to depend heavily on $H(\cdot)$, it is essentially the same when $\varepsilon \rightarrow 0$. More precisely, given another hyperplane selection $H^{\prime}(\cdot)$, there are constants $c_{1}$ and $c_{2}$ (independent of $\varepsilon$ ) such that for all small enough $\varepsilon>0$

$$
S\left(T, H, c_{1} \varepsilon\right) \subset S\left(T, H^{\prime}, \varepsilon\right) \subset S\left(T, H, c_{2} \varepsilon\right) .
$$

This can be proved by induction in an obvious way. We will write $S(T, \varepsilon)$ for $S_{P}(T, H, \varepsilon)$ as we think of $P$ and $H(\cdot)$ as being fixed.

The notation (vert $P_{n}$ in $A$ ) will denote the number of vertices of $P_{n}$ in $A \subset \mathbb{R}^{d}$. The vertices of $P_{n}$ are concentrated in the simplices $S(T, \varepsilon)$ with $\varepsilon=(\log n)^{-1}$ in the following sense:

Theorem 3. Let $P \in \mathscr{K}_{1}^{d}, d \geq 2$, and set $\varepsilon=(\log n)^{-1}$. Then

$$
E\left(\text { vert } P_{n} \text { in } P \backslash \bigcup_{T} S(T, \varepsilon)\right) \leq \operatorname{const}(P) \log ^{d \sim 2} n \log \log n \text {. }
$$

This is one of the results needed for Theorem 2: The other one is more difficult to prove, and we like to call it "independence of shape".

Theorem 4. Let $P \in \mathscr{K}_{1}^{d}, d \geq 2$, and set $\varepsilon=(\log n)^{-1}$. Then for any tower $T$ of $P$

$$
E\left(\text { vert } P_{n} \text { in } S(T, \varepsilon)\right)=\frac{1}{(d+1)^{d-1}(d-1) !} \log ^{d-1} n+O\left(\log ^{d-2} n \log \log n\right) .
$$

This shows that $S(T, \varepsilon)$ contains essentially the same number of vertices of $P_{n}$ no matter what the shape of $P$ is. Actually, we will prove that $E$ (vert $P_{n}$ in $S(T, \varepsilon)$ ) is the same for all $T$ independently of $P$ up to $O\left(\log ^{d-2} n \log \log n\right)$. Then this number will be implied from the result of Affentranger and Wieacker.

Theorems 3 and 4 state that the vertices of $P_{n}$ are concentrated in $\cup_{T} S(T, \varepsilon)$ and that their number in any particular simplex $S(T, \varepsilon)$ is essentially independent of the shape of $P$. This is true not only for the vertices but for the $k$-dimensional faces of $P_{n}$ as well. Let us write $f_{k}(P)$ for the number of $k$-dimensional faces of the polytope $P$. Then the following analogue of (1.6) holds.

Theorem 5. For a polytope $P \in \mathscr{K}^{d}$ and $k=0,1, \ldots, d-1$

$$
E\left(f_{k}\left(P_{n}\right)\right)=C(d, k) T(P) \log ^{d-1} n+O\left(\log ^{d-2} n \log \log n\right)
$$

where $C(d, k)$ is a constant depending only on $d$ and $k$. 
The proof of this theorem is based on statements analogous to Theorems 3 and 4. As it is quite technical and does not require new ideas, we will not present it here.

It can be seen from the work of Affentranger and Wieacker [AW] that

$$
\begin{aligned}
& C(d, 0)=\frac{d^{d-1}}{((d-1) !)^{2}} M_{2}\left(\Delta_{d-1}\right), \\
& C(d, d-1)=\frac{d^{d-2}}{((d-1) !)^{2}} M_{1}\left(\Delta_{d-1}\right),
\end{aligned}
$$

where $M_{k}\left(\Delta_{d-1}\right)$ denotes the $k$-th moment of the volume of the convex hull of $d$ random points in a simplex $\Delta_{d-1} \in \mathscr{E}_{1}^{d-1}$. Due to Reed [Re],

$$
M_{2}\left(\Delta_{d-1}\right)=\frac{(d-1) !}{d^{d-1}(d+1)^{d-1}},
$$

whence $C(d, 0)$ follows as stated in (1.6). However, $M_{1}\left(\Delta_{d-1}\right)$ is not known for $d \geq 5$. $\left(M_{1}\left(\Delta_{1}\right)=\frac{1}{3}, M_{1}\left(\Delta_{2}\right)=\frac{1}{12}\right.$, and it was recently proved by Buchta and Reitzner $[\mathrm{BR}]$ that $M_{1}\left(\Delta_{3}\right)=\frac{13}{720}-\frac{\pi^{2}}{15015}$.)

Since $P_{n}$ is simplicial with probability 1 , for $j=-1,0, \ldots, d-2$

$$
\sum_{k=j}^{d-1}(-1)^{k}\left(\begin{array}{l}
k+1 \\
j+1
\end{array}\right) C(d, k)=(-1)^{d-1} C(d, j)
$$

with $C(d,-1)=0$, other than in the usual Dehn-Sommerville equations where the corresponding value is 1 . (Euler's theorem $\sum_{k=0}^{d-1}(-1)^{k} f_{k}=1-(-1)^{d}$ corresponds to $\sum_{k=0}^{d-1}(-1)^{k} C(d, k)=0$.) For example, in the three-dimensional case, (1.12) and $C(3,0)=\frac{1}{32}$ imply $C(3,1)=\frac{3}{32}, C(3,2)=\frac{1}{16}$. (The resulting expressions for $E\left(f_{k}\left(P_{n}\right)\right)$ can be simplified by observing that $T(P)$ is four times the number of edges for every three-dimensional polytope $P$.)

The results of this paper were announced in Bárány, Buchta $[B B]$. For further information about the convex hull of random points and related topics see the section "Random points in a convex body" in the work of Weil and Wieacker [WW] as well as the surveys of Affentranger [Af2], Schneider [Schn], and Buchta [Bu3]. Interesting remarks are also contained in the section "Random polygons and polyhedra" of a new book on unsolved problems in geometry [CFG].

\section{Notation, definitions, further results}

Given a convex body $K \in \mathscr{K}^{d}$ and $\theta>0$, the Macbeath region with centre $x \in K$ is defined as

$$
M(x, \theta)=M_{K}(x, \theta)=x+\theta[(K-x) \cap(x-K)] .
$$

Sometimes we will write $M(x)$ instead of $M(x, 1)$. Macbeath regions were studied in [Ma], [ELR], [BáLa], and [Bá]. Define $u=u_{K}: K \rightarrow \mathbb{R}$ by

$$
u(x)=\operatorname{vol} M_{K}(x) .
$$

Another function of interest is $v=v_{K}: K \rightarrow \mathbb{R}$ which is defined by 


$$
v(x)=\min \left\{\operatorname{vol}\left(K \cap H^{+}\right): x \in H^{+}, H^{+} \text {a halfspace }\right\} \text {. }
$$

It is deduced in [BáLa] that $u(x) \leq 2 v(x)$ for every $x \in K$ and $v(x) \leq(3 d)^{d} u(x)$ if $u(x)$ or $v(x)$ is sufficiently small.

We write $K(u \leq \varepsilon)$ for $\{x \in K: u(x) \leq \varepsilon\}$; the sets $K(u \geq \varepsilon), K(v \leq \varepsilon)$, and $K(v \geq \varepsilon)$ are defined analogously. Macbeath proved that $K(u \geq \varepsilon)$ is convex, see Sections 7 and 11 of [Ma]. Obviously $K(v \geq \varepsilon)$ is convex because it is the intersection of closed halfspaces.

The main result of [BáLa] states that $E(K, n)$ is "essentially the same" as vol $K\left(v \leq \frac{1}{n}\right)$. Precisely, there are constants $c_{1}(d)$ and $c_{2}(d)$ such that

$$
c_{1}(d) E(K, n) \leq \operatorname{vol} K\left(v \leq \frac{1}{n}\right) \leq c_{2}(d) E(K, n)
$$

for $K \in \mathscr{F}_{1}^{d}$ and $n \geq d+1$. Moreover, vol $K\left(v \leq \frac{1}{n}\right)$ and $\operatorname{vol} K\left(u \leq \frac{1}{n}\right)$ are essentially the same, too.

In the case of a polytope we can prove a formula similar to (1.4):

Theorem 6. Let $P \in \mathscr{F}_{1}^{d}$ be a polytope, $d \geq 2$. Then

$$
\operatorname{vol} P(u \leq \varepsilon)=\frac{T(P)}{2^{d} d !(d-1) !} \varepsilon \log ^{d-1} \frac{1}{\varepsilon}+O\left(\varepsilon \log ^{d-2} \frac{1}{\varepsilon}\right) \text {. }
$$

Albeit much simpler than Theorem 2 this will be quite useful. Analogously one can show

$$
\operatorname{vol} P(v \leq \varepsilon)=\frac{T(P)}{d^{d}(d-1) !} \varepsilon \log ^{d-1} \frac{1}{\varepsilon}+O\left(\varepsilon \log ^{d-2} \frac{1}{\varepsilon}\right) \text {. }
$$

This was first proved by Schütt [Schui], we found it independently.

The assumption vol $K=1$ or vol $P=1$ in the theorems is made for convenience rather than necessity. What is really needed is vol $K>0$, and we will have to consider convex bodies with vol $K \neq 1$ as well. In this case it is better to take

$$
\frac{\operatorname{vol} K(u \leq \varepsilon \operatorname{vol} K)}{\operatorname{vol} K}
$$

instead of vol $K(u \leq \varepsilon)$ because it is affinely invariant. Precisely, let $L: \mathbb{R}^{d} \rightarrow \mathbb{R}^{d}$ be a nondegenerate affine transformation and $K \in \mathscr{K}^{d}$. Then, clearly,

$$
\frac{\operatorname{vol} K\left(u_{K} \leq \varepsilon \operatorname{vol} K\right)}{\operatorname{vol} K}=\frac{\operatorname{vol} L K\left(u_{L K} \leq \varepsilon \operatorname{vol} L K\right)}{\operatorname{vol} L K} .
$$

We mention further that $E$ (vert $K_{n}$ ) does not depend on the volume of $K$. But Efron's identity (1.5) has to be modified:

$$
E\left(\text { vert } K_{n+1}\right)=\frac{n+1}{\operatorname{vol} K} E(K, n) \text { when } K \in \mathscr{K}^{d} \text {. }
$$

Assume $P \in \mathscr{K}^{d}$ is a polytope and let $T$ be one of its towers. This will define parameters $\tau_{0}(z), \tau_{1}(z), \ldots, \tau_{d-1}(z)$ for $z \in P$ in the following way. We use induction, so when $d=1, \tau_{0}(z)$ is the distance of $z$ from the vertex defining $T$. When $d>1, \tau_{0}(z)$ is defined (cf. (1.7) and (1.8)) by

$$
z \in H_{0}\left(\tau_{0}(z)\right) .
$$


Recall the definitions of $Q, T_{Q}, S_{Q}\left(T_{Q}, \varepsilon\right)$ from $(1.7),(1.8),(1.9),(1.10),(1.11)$. Set

$$
z_{Q}:=t_{0} \tau_{0}^{-1}(z) z \in Q
$$

Define now for $i=1,2, \ldots, d-1$

$$
\tau_{i}(z)=\tau_{i-1}\left(z_{Q}\right)
$$

where the parameter $\tau_{i-1}\left(z_{Q}\right)$ is meant in $Q$ with respect to the tower $T_{Q}$. With this definition we have

$$
z \in S(T, \varepsilon) \text { if and only if } \tau_{0}(z) \leq \varepsilon \text { and } z_{Q} \in S_{Q}\left(T_{Q}, \varepsilon\right)
$$

and, further,

$$
z \in S(T, \varepsilon) \text { if and only if } \tau_{i}(z) \leq \varepsilon \quad(i=0,1, \ldots, d-1) .
$$

Clearly, for $\alpha>0$ and $z \in P$

$$
\tau_{0}(\alpha z)=\alpha \tau_{0}(z)
$$

but

$$
\tau_{i}(\alpha z)=\tau_{i}(z) \quad(i=1, \ldots, d-1) .
$$

In the proof of Theorem 4 we will need the following notation. Again, $P$ is a polytope and $T=\left(F_{0}, F_{1}, \ldots, F_{d-1}\right)$ a tower of $P$. For $\phi_{0}, \phi_{1}, \ldots, \phi_{d-1}>0$ define

$$
\begin{aligned}
P\left(\tilde{\phi}_{i}\right) & =P\left(\phi_{0}, \ldots, \phi_{i}\right) \\
& =P\left(\phi_{0}, \ldots, \phi_{i} ; F_{0}, \ldots, F_{i}\right) \\
& =\left\{z \in P: \tau_{j}(z) \leq \phi_{j}(j=0, \ldots, i)\right\} .
\end{aligned}
$$

In particular, if $\varphi_{0}=\varphi_{1}=\cdots=\varphi_{d-1}=\varepsilon$, then

$$
P\left(\bar{\varphi}_{d-1}\right)=S(T, \varepsilon) \text {. }
$$

Moreover, we put

$$
P\left(\bar{\phi}_{i-1}\right)=P \text { when } i=0 \text {, }
$$

and we set for $i=0,1, \ldots, d-1$

$$
\begin{aligned}
& P\left(\bar{\phi}_{i-1}, \tau_{i} \geq \phi_{i}\right) \\
& =P\left(\phi_{0}, \ldots, \phi_{i-1}, \tau_{i} \geq \phi_{i}\right) \\
& =\left\{z \in P\left(\bar{\phi}_{i-1}\right): \tau_{i}(z) \geq \phi_{i}\right\} .
\end{aligned}
$$

Notice that for $i \geq 1$

$$
P\left(\bar{\phi}_{i-1}, \tau_{i} \geq \phi_{i}\right)=\operatorname{cone} Q\left(\phi_{1}, \ldots, \phi_{i-1}, \tau_{i-1}^{(Q)} \geq \phi_{i}\right) \cap H_{0}\left(0, \phi_{0}\right)
$$

where $\tau_{i-1}^{(Q)}$ is the $(i-1)^{\text {st }}$ parameter induced in $Q$ by the tower $T$.

Finally, we define

$$
\operatorname{ray}(x, y)=\{x+t(y-x): t \geq 0\}
$$

and we set

$$
u(x, y)=\max \{u(z): z \in \operatorname{aff}(x, y)\}
$$


where $u: \mathbb{R}^{d} \rightarrow \mathbb{R}$ and aff $(x, y)$ denotes the affine hull of $x, y \in \mathbb{R}^{d}$.

We will use the notation const $(P)$ for different constants. As we think that the hyperplane selection $H(\cdot)$ is given together with the polytope $P$, we will write const $(P)$ instead of $\operatorname{const}(P, H)$.

\section{Auxiliary results}

For $0 \leq \varepsilon \leq 1$

$$
\operatorname{vol}\left\{x \in \mathbb{R}^{d}: \prod_{i=1}^{d} x_{i} \leq \varepsilon, 0 \leq x_{i} \leq 1(i=1, \ldots, d)\right\}=\varepsilon \sum_{i=0}^{d-1} \frac{1}{i !} \log ^{i} \frac{1}{\varepsilon} .
$$

This follows, e.g., from (3.5) and (8.1) in Chapter I of [Fe].

Assume now that $P$ is a polytope with a fixed tower $T$ whose starting vertex is the origin. Then

$$
u_{P}(x)=\int_{\tau_{0}(x)-\tau_{0}}^{\tau_{0}(x)+\tau_{0}} \operatorname{vol}_{d-1}\left[M_{P}(x) \cap H_{0}(t)\right] d t
$$

where $\tau_{0} \geq 0$ is defined as the largest $t$ for which the section $M_{P}(x) \cap H_{0}\left(\tau_{0}(x)-t\right)$ is nonempty. It is easy to see that the central section $M_{P}(x) \cap H_{0}\left(\tau_{0}(x)\right)$ coincides with $M_{Q\left(\tau_{0}(x)\right)}(x)$. Since $M_{P}(x)$ is centrally symmetric with centre $x$, the largest volume section is the central one. Then (3.2) implies

$$
u_{P}(x) \leq 2 \tau_{0} u_{Q\left(\tau_{0}(x)\right)}(x)
$$

On the other hand,

$$
\begin{aligned}
u_{P}(x) & =2 \int_{\tau_{0}(x)-\tau_{0}}^{\tau_{0}(x)} \operatorname{vol}_{d-1}\left[M_{P}(x) \cap H_{0}(t)\right] d t \\
& \geq 2 \int_{\tau_{0}(x)-\tau_{0}}^{\tau_{0}(x)}\left(\frac{t-\tau_{0}(x)+\tau_{0}}{\tau_{0}}\right)^{d-1} \operatorname{vol}_{d-1} M_{Q\left(\tau_{0}(x)\right)}(x) d t \\
& =\frac{2 \tau_{0}}{d} u_{Q\left(\tau_{0}(x)\right)}(x) .
\end{aligned}
$$

We will often use (3.3) and (3.4) when $\tau_{0}=\tau_{0}(x)$. This happens if $x$ is close enough to the vertex of $T$, for instance, if the vertex of $T$ is the only vertex of $P$ lying in the slab $H_{0}\left(0,2 \tau_{0}(x)\right)$.

Assume now that $K \in \mathscr{K}^{d}$ with vol $K=q$. It can be seen from the proof of Theorem 1 in [BáLa] that

$$
\operatorname{Prob}\left(x \notin K_{n}\right) \leq 2 \sum_{i=0}^{d-1}\left(\begin{array}{c}
n \\
i
\end{array}\right)\left(\frac{u(x)}{2 q}\right)^{i}\left(1-\frac{u(x)}{2 q}\right)^{n-i}
$$

where Prob is meant with $x \in K$ fixed and $K_{n}$ the random polytope in $K$ varying. 
Before stating the first of three lemmata needed in the proof of Theorem 4, we mention a result of Macbeath: Let $L$ be a convex compact subset of $K$ containing interior points of $K$. Then, according to Lemma 7.1 in [Ma], the maximum value of $u_{K}$ in $L$ is attained at a unique point of $L$.

Lemma 1. Assume $K \in \mathscr{F}^{d}$, and $a$ and b are points on the boundary of $K$ such that aff $(a, b)$ contains interior points of $K$. Let $c$ be the point where $u$ takes its maximum value on aff $(a, b)$. Then, if $u(c)$ is sufficiently small,

$$
\frac{\|a-c\|}{\|b-c\|} \leq(3 d)^{d+2} \text {. }
$$

Lemma 1 says that if $H$ is a hyperplane and $u(c)=\max \{u(x): x \in H\}$ with $c \in H$, then $c$ is a " $(3 d)^{d+2}$-central" point of the section $K \cap H$. Similarly, the $v$-maximal point on $H$ is the centre of gravity of $K \cap H$ (cf., e.g., the proof of Lemma 4 in [ELR]), whence it is " $(d-1)$-central".

Lemma 2. Assume $P \in \mathscr{K}_{1}^{d}, T$ is a tower of $P, \varphi_{0}=\varphi_{1}=\ldots=\varphi_{d-1}=\left(\log \frac{1}{\varepsilon}\right)^{-1}$ with $\varepsilon>0$ small enough, $\phi_{0}, \phi_{1}, \ldots, \phi_{d-1}>0$ are constants, $\theta \geq 1$. Then, for $i=0,1, \ldots, d-1, x \in P\left(\vec{\varphi}_{i}\right)$ implies

$$
\begin{array}{r}
\operatorname{vol}\left[P\left(\bar{\phi}_{i-1}, \tau_{i} \geq \phi_{i}\right) \cap M_{P\left(\bar{\phi}_{i-1}\right)}(x, \theta)\right] \\
\leq \operatorname{const}(P) \tau_{i}(x) \operatorname{vol} M_{P\left(\bar{\phi}_{i-1}\right)}(x, \theta) .
\end{array}
$$

Lemma 3. Assume, again, $P \in \mathscr{R}_{1}^{d}, T$ is a tower of $P, \varphi_{0}=\varphi_{1}=\ldots=\varphi_{d-1}=$ $\left(\log \frac{1}{\varepsilon}\right)^{-1}$ with $\varepsilon>0$ small enough, $\phi_{0}, \phi_{1}, \ldots, \phi_{d-1}>0$ are constants. Then, for $i=0,1, \ldots, d-1$,

$$
\begin{aligned}
\operatorname{meas} & \left\{(x, y) \in P\left(\bar{\varphi}_{i}\right) \times P\left(\bar{\phi}_{i-1}, \tau_{i} \geq \phi_{i}\right): u_{P\left(\bar{\phi}_{i-1}\right)}(x, y) \leq \varepsilon\right\} \\
& \leq \operatorname{const}(P) \varepsilon^{2} \log ^{d-2} \frac{1}{\varepsilon} \log \log \frac{1}{\varepsilon}
\end{aligned}
$$

where meas is the product of the Lebesgue measures on $\mathbb{R}^{d} \times \mathbb{R}^{d}$.

The proofs of the lemmata are given in Section 8. In Section 7 we deduce Theorem 1 from Theorem 2. The proof of Theorem 2 consists in proving Theorems 3 and 4 which will be done in Sections 5 and 6. Theorem 6, or rather its proof, turns out to be an important tool for the proofs of Theorem 3 and 4 , so we start with Theorem 6.

\section{Proof of Theorem 6}

For a vertex $v \in P$ define $H_{v}=H(\{v\})$ and write $H_{v}(0, \varphi)$ for the slab between $H_{v}$ and $H_{v}(\varphi)$. Put $A(\varphi)=P \backslash \cup_{v} H_{v}(0, \varphi)$. As a first step in the proof we show

$$
\operatorname{vol}[P(u \leq \varepsilon) \cap A(\varphi)] \leq \operatorname{const}(P) \varepsilon \log ^{d-2} \frac{1}{\varepsilon} \log \frac{1}{\varphi}
$$

provided $\varphi^{d} \geq \operatorname{const}(P) \varepsilon$. (4.1) means that the essential part of $P(u \leq \varepsilon)$ is concentrated near the vertices of $P$. 
When $d=1$ and $\varphi \geq \varepsilon / 2$, the left hand side of (4.1) equals 0 . For $d \geq 2$ let $\Delta_{1}, \ldots, \Delta_{m}$ be simplices forming a triangulation of $P$ that uses vertices of $P$ only. Clearly,

$$
P(u \leq \varepsilon) \subset \bigcup_{i=1}^{m} \Delta_{i}\left(u_{\Delta i} \leq \varepsilon\right)
$$

Now for a simplex $\Delta \in \mathscr{K}_{1}^{d}$ with hyperplane selection $H(\cdot)$ one can show that

$$
\begin{aligned}
& \operatorname{vol}\left\{x \in \Delta: u_{\Delta}(x) \leq \varepsilon, x \notin \underset{v \text { a vertex of } \Delta}{\bigcup_{v}} H_{v}(0, \varphi)\right\} \\
& \quad \leq \operatorname{const}(d) \varepsilon \log ^{d-2} \frac{1}{\varepsilon} \log \frac{1}{\varphi}
\end{aligned}
$$

provided $\varphi^{d} \geq$ const $(d) \varepsilon$. The proof of this is a routine calculation using (3.1) and is, therefore, omitted. See [Dw], [AW] for a similar computation.

Using an affine transformation carrying $\Delta_{i}$ into $\Delta$ we get by (2.2)

$$
\begin{aligned}
\operatorname{vol}\left\{x \in \Delta_{i}: u_{\Delta_{i}}(x) \leq \varepsilon, x \notin \bigcup_{v \text { a vertex of } \Delta_{i}} H_{v}(0, \varphi)\right\} \\
\quad \leq \operatorname{vol} \Delta_{i} \operatorname{vol}\left\{x \in \Delta: u_{\Delta}(x) \leq \frac{\varepsilon}{\operatorname{vol} \Delta_{i}}, x \notin \bigcup_{v \text { a vertex of } \Delta} H_{v}\left(0, \frac{\varphi}{\left(\operatorname{vol} \Delta_{i}\right)^{1 / d}}\right)\right\} \\
\quad \leq \operatorname{vol} \Delta_{i} \operatorname{const}(d) \frac{\varepsilon}{\operatorname{vol} \Delta_{i}} \log ^{d-2} \frac{\operatorname{vol} \Delta_{i}}{\varepsilon} \log \frac{\left(\operatorname{vol} \Delta_{i}\right)^{1 / d}}{\varphi} \\
\leq \operatorname{const}(d) \varepsilon \log ^{d-2} \frac{1}{\varepsilon} \log \frac{1}{\varphi}
\end{aligned}
$$

provided $\left(\frac{\varphi}{\left(\operatorname{vol} \Delta_{i}\right)^{1 / d}}\right)^{d} \geq \operatorname{const}(d) \frac{\varepsilon}{\operatorname{vol} \Delta_{i}}$, i.e. $\varphi^{d} \geq \operatorname{const}(d) \varepsilon$. Summing this for all $\Delta_{i}$ we get (4.1).

It is helpful for the second step in the proof to notice that analogous arguments easily give

$$
\text { vol } P(u \leq \varepsilon) \leq \operatorname{const}(P) \varepsilon \log ^{d-1} \frac{1}{\varepsilon} \text {. }
$$

This second step consists in showing that $P(u \leq \varepsilon)$ is concentrated in the union of the simplices $S(T, \varphi)$. Setting now $B(\varphi)=P \backslash \cup_{T} S(T, \varphi)$ we claim

$$
\operatorname{vol}[P(u \leq \varepsilon) \cap B(\varphi)] \leq \operatorname{const}(P) \varepsilon \log ^{d-2} \frac{1}{\varepsilon} \log \frac{1}{\varphi}
$$

provided $\varphi^{d} \geq \operatorname{const}(P) \varepsilon$. We prove (4.4) by induction on $d$. The case $d=1$ is trivial. The case $d=2$ which needs special consideration is quite simple and is left to the reader.

Since $B(\varphi) \supset A(\varphi)=P \backslash \cup_{v} H_{v}(0, \varphi)$ we have

$$
P(u \leq \varepsilon) \cap B(\varphi)=[P(u \leq \varepsilon) \cap A(\varphi)] \cup \bigcup_{v}\left[P(u \leq \varepsilon) \cap B(\varphi) \cap H_{v}(0, \varphi)\right],
$$




$$
\begin{aligned}
& \operatorname{vol}[P(u \leq \varepsilon) \cap B(\varphi)] \\
& \quad \leq \operatorname{vol}[P(u \leq \varepsilon) \cap A(\varphi)]+\sum_{v} \operatorname{vol}\left[P(u \leq \varepsilon) \cap B(\varphi) \cap H_{v}(0, \varphi)\right]
\end{aligned}
$$

We will estimate

$$
\begin{aligned}
O(v) & :=\operatorname{vol}\left[P(u \leq \varepsilon) \cap B(\varphi) \cap H_{v}(0, \varphi)\right] \\
& =\operatorname{vol}\left\{x \in P: u(x) \leq \varepsilon, x \notin \bigcup S(T, \varphi), x \in H_{v}(0, \varphi)\right\}
\end{aligned}
$$

separately for each vertex $v$. We suppose $v=0$, again. Assume $\varphi$ is so small that the only vertex lying in $H_{0}(0,2 \varphi)$ is $v=0$. Consequently, for $x \in P \cap H_{0}(0, \varphi)$

$$
M_{P}(x, 1)=M_{\text {conv }(Q \cup\{0\})}(x, 1)
$$

where $Q$ is defined in (1.8), cf. (1.7) as well. Then

$$
O(v)=\int_{0}^{\varphi} \operatorname{vol}_{d-1}\left\{x \in Q(t): u_{P}(x) \leq \varepsilon, x \notin \bigcup S(T, \varepsilon)\right\} d t .
$$

We estimate the integrand in (4.6) using successively (3.4), (2.2), the fact that $\operatorname{vol}_{d-1} Q(t)=c_{1}(Q) t^{d-1}$, and the induction hypothesis

$$
\begin{gathered}
\operatorname{vol}_{d-1}\left\{x \in Q: u_{Q}(x) \leq \varepsilon, x \notin \bigcup S_{Q}\left(T_{Q}, \varphi\right)\right\} \\
\leq \operatorname{const}(Q) \varepsilon \log ^{d-3} \frac{1}{\varepsilon} \log \frac{1}{\varphi}
\end{gathered}
$$

provided $\varphi^{d-1} \geq c_{2}(Q) \varepsilon ;$ cf. (4.4) and (1.9). Thus we obtain

$$
\begin{aligned}
\operatorname{vol}_{d-1} & \left\{x \in Q(t): u_{P}(x) \leq \varepsilon, x \notin \bigcup S(T, \varphi)\right\} \\
& \leq \operatorname{vol}_{d-1}\left\{x \in Q(t): u_{Q(t)}(x) \leq \frac{d \varepsilon}{2 t}, x \notin \bigcup S(T, \varphi)\right\} \\
& =\frac{\operatorname{vol}_{d-1} Q(t)}{\operatorname{vol} Q} \operatorname{vol}_{d-1}\left\{x \in Q: u_{Q}(x) \leq \frac{d \varepsilon \operatorname{vol}_{d-1} Q}{2 t \operatorname{vol}_{d-1} Q(t)}, x \notin \bigcup S_{Q}\left(T_{Q}, \varphi\right)\right\} \\
& =c_{1}(Q) t^{d-1} \operatorname{vol}_{d-1}\left\{x \in Q: u_{Q}(x) \leq \frac{d \varepsilon}{2 c_{1}(Q) t^{d}}, x \notin \bigcup S_{Q}\left(T_{Q}, \varphi\right)\right\} \\
& \leq c_{1}(Q) t^{d-1} \operatorname{const}(Q) \frac{d \varepsilon}{2 c_{1}(Q) t^{d}} \log \frac{d-3}{\frac{2 c_{1}(Q) t^{d}}{d \varepsilon}} \log \frac{1}{\varphi} \\
& =\operatorname{const}(Q) \frac{\varepsilon}{t} \log ^{d-3} \frac{2 c_{1}(Q) t^{d}}{d \varepsilon} \log \frac{1}{\varphi}
\end{aligned}
$$

provided $\varphi^{d-1} \geq c_{2}(Q) \frac{d \varepsilon}{2 c_{1}(Q) t^{d}}$ and $\frac{d \varepsilon}{2 c_{1}(Q) t^{d}} \leq 1$. Define $t_{2}$ and $t_{1}$ as the smallest values $t>0$ such that these inequalities hold, i.e.

$$
t_{2}^{d}=c_{2}(Q) \frac{d \varepsilon}{2 c_{1}(Q) \varphi^{d-1}} \text { and } t_{1}^{d}=\frac{d \varepsilon}{2 c_{1}(Q)}
$$

Notice that $t_{2} \geq t_{1}$ as $\frac{c_{2}(Q)}{\varphi^{d-1}} \geq 1$. 
We apply (4.8) when $t_{2} \leq t \leq \varphi$. Observing $\frac{2 c_{1}(Q) \varphi^{d}}{d} \leq 1$ (as the volume of $P$ is 1) we see that

$$
\begin{aligned}
& \int_{t_{2}}^{\varphi} \operatorname{vol}_{d-1}\left\{x \in Q(t): u_{P}(x) \leq \varepsilon, x \notin \bigcup S(T, \varphi)\right\} d t \\
& \quad \leq \int_{t_{2}}^{\varphi} \operatorname{const}(Q) \frac{\varepsilon}{t} \log ^{d-3} \frac{2 c_{1}(Q) t^{d}}{d \varepsilon} \log \frac{1}{\varphi} d t \\
& \quad=\operatorname{const}(Q) \frac{1}{d(d-2)} \varepsilon\left(\log ^{d-2} \frac{2 c_{1}(Q) \varphi^{d}}{d \varepsilon}-\log ^{d-2} \frac{c_{2}(Q)}{\varphi^{d-1}}\right) \log \frac{1}{\varphi} \\
& \quad \leq \operatorname{const}(Q) \varepsilon \log ^{d-2} \frac{1}{\varepsilon} \log \frac{1}{\varphi} .
\end{aligned}
$$

For $t_{1} \leq t \leq t_{2}$ we use

$$
\operatorname{vol}_{d-1}\left\{x \in Q: u_{Q}(x) \leq \varepsilon\right\} \leq \operatorname{const}(Q) \varepsilon \log ^{d-2} \frac{1}{\varepsilon}
$$

instead of (4.7); cf. (4.3). (Applying (4.3) can be avoided if the whole theorem is proved by induction.) Then

$$
\begin{aligned}
& \int_{t_{1}}^{t_{2}} \operatorname{vol}_{d-1}\left\{x \in Q(t): u_{P}(x) \leq \varepsilon, x \notin \bigcup S(T, \varphi)\right\} d t \\
& \leq \int_{t_{1}}^{t_{2}} \operatorname{vol}_{d-1}\left\{x \in Q(t): u_{P}(x) \leq \varepsilon\right\} d t \\
& \leq \int_{t_{1}}^{t_{2}} c_{1}(Q) t^{d-1} \operatorname{vol}_{d-1}\left\{x \in Q: u_{Q}(x) \leq \frac{d \varepsilon}{2 c_{1}(Q) t^{d i}}\right\} d t \\
& \leq \int_{t_{1}}^{t_{2}} c_{1}(Q) t^{d-1} \operatorname{const}(Q) \frac{d \varepsilon}{2 c_{1}(Q) t^{d}} \log ^{d-2} \frac{2 c_{1}(Q) t^{d}}{d \varepsilon} d t \\
& =\int_{t_{1}}^{t_{2}} \operatorname{const}(Q) \frac{\varepsilon}{t} \log ^{d-2} \frac{2 c_{1}(Q) t^{d}}{d \varepsilon} d t \\
& =\operatorname{const}(Q) \frac{1}{d(d-1)} \varepsilon \log ^{d-1} \frac{c_{2}(Q)}{\varphi^{d-1}} \\
& \leq \operatorname{const}(Q) \varepsilon \log ^{d-2} \frac{1}{\varepsilon} \log \frac{1}{\varphi}
\end{aligned}
$$

since $1 \leq \frac{c_{2}(Q)}{\varphi^{d-1}} \leq \frac{1}{\varepsilon}$.

Finally, for $0 \leq t \leq t_{1}$ 


$$
\begin{aligned}
& \int_{0}^{t_{1}} \operatorname{vol}_{d-1}\left\{x \in Q(t): u_{P}(x) \leq \varepsilon, x \notin \bigcup S(T, \varphi)\right\} d t \\
& \quad \leq \int_{0}^{t_{1}} \operatorname{vol}_{d-1} Q(t) d t=\int_{0}^{t_{1}} c_{1}(Q) t^{d-1} d t=\frac{\varepsilon}{2} .
\end{aligned}
$$

To summarize, we conclude that

$$
O(v) \leq \operatorname{const}(Q) \varepsilon \log ^{d-2} \frac{1}{\varepsilon} \log \frac{1}{\varphi} .
$$

Because of (4.5), this together with (4.1) proves (4.4).

As a third and last step in the proof we compute vol $[P(u \leq \varepsilon) \cap S(T, \varphi)]$. We do this first when $P=C$, the unit cube in $\mathbb{R}^{d}$. In this case, by symmetry, $C(u \leq \varepsilon) \cap S(T, \varphi)$ is the same for all towers $T$ of $C$. On the other hand,

$$
u_{C}(x)=2^{d} x_{1} \ldots x_{d}
$$

for those $x=\left(x_{1}, \ldots, x_{d}\right) \in C$ which satisfy $0 \leq x_{i} \leq \frac{1}{2}(i=1, \ldots, d)$. A routine computation similar to the one needed for (4.2) gives

$$
\begin{gathered}
\operatorname{vol}\left\{x \in C: u_{C}(x) \leq \varepsilon, x_{i} \leq \frac{1}{2}(i=1, \ldots, d)\right\} \\
=\frac{1}{2^{d}(d-1) !} \varepsilon \log ^{d-1} \frac{1}{\varepsilon}+O\left(\varepsilon \log ^{d-2} \frac{1}{\varepsilon}\right) .
\end{gathered}
$$

Since there are $d$ ! towers and so $d$ ! simplices $S(T, \varphi)$ starting with $F_{0}=\{0\}$, we get

$$
\operatorname{vol}\left[C\left(u_{C} \leq \varepsilon\right) \cap S(T, \varphi)\right]=\frac{1}{2^{d} d !(d-1) !} \varepsilon \log ^{d-1} \frac{1}{\varepsilon}+O\left(\varepsilon \log ^{d-2} \frac{1}{\varepsilon} \log \frac{1}{\varphi}\right),
$$

where we used (4.4) with $P=C$ as well.

Assume now $P$ is a polytope and $T$ is one of its towers. Then one can find two parallelepipeda $C_{1}$ and $C_{2}$ with towers $T_{1}$ and $T_{2}$ so that $S_{P}(T, \varphi)=S_{C_{1}}\left(T_{1}, \varphi\right)=$ $S_{C_{2}}\left(T_{2}, \varphi\right)$ and that for $x$ close enough to the origin

$$
\begin{aligned}
& x \in C_{1} \text { implies } x \in P \text { and } \\
& x \in P \text { implies } x \in C_{2} .
\end{aligned}
$$

Now if $x \in S(T, \varphi)$ and $\varphi$ is small enough, then $x$ is close to the origin and so

$$
M_{C_{1}}(x) \subset M_{P}(x) \subset M_{C_{2}}(x) .
$$

Consequently $u_{C_{1}}(x) \leq u_{P}(x) \leq u_{C_{2}}(x)$. We know from (4.9) and (2.2) that for $i=1,2$

$$
\operatorname{vol}\left[C_{i}\left(u_{C_{i}} \leq \varepsilon\right) \cap S\left(T_{i}, \varphi\right)\right]=\frac{1}{2^{d} d !(d-1) !} \varepsilon \log ^{d-1} \frac{1}{\varepsilon}+O\left(\varepsilon \log ^{d-2} \frac{1}{\varepsilon} \log \frac{1}{\varphi}\right)
$$

proving that

$$
\operatorname{vol}\left[P\left(u_{P} \leq \varepsilon\right) \cap S(T, \varphi)\right]=\frac{1}{2^{d} d !(d-1) !} \varepsilon \log ^{d-1} \frac{1}{\varepsilon}+O\left(\varepsilon \log ^{d-2} \frac{1}{\varepsilon} \log \frac{1}{\varphi}\right) .
$$


Finally, summing (4.10) for all the towers and using (4.4) gives

$$
\operatorname{vol} P(u \leq \varepsilon)=\frac{T(P)}{2^{d} d !(d-1) !} \varepsilon \log ^{d-1} \frac{1}{\varepsilon}+O\left(\varepsilon \log ^{d-2} \frac{1}{\varepsilon} \log \frac{1}{\varphi}\right)
$$

provided $\varphi^{d} \geq \operatorname{const}(P) \varepsilon$. This certainly holds when $\varphi$ is a suitable constant and $\varepsilon>0$ small enough, proving the theorem.

\section{Proof of Theorem 3}

Assume $A \subset P$ is measurable. Set $X_{n}=\left\{x_{1}, \ldots, x_{n}\right\}$. Clearly,

$$
\begin{aligned}
E\left(\operatorname{vert} P_{n} \text { in } A\right) & =\sum_{i=1}^{n} \operatorname{Prob}\left(x_{i} \in A, x_{i} \notin \operatorname{conv}\left(X_{n} \backslash\left\{x_{i}\right\}\right)\right) \\
& =n \int_{x \in A} \operatorname{Prob}\left(x \notin P_{n-1}\right) d x .
\end{aligned}
$$

Here $\operatorname{Prob}\left(x \notin P_{n-1}\right)$ is meant with $x$ fixed and $P_{n-1}=\operatorname{conv} X_{n-1}$, a random polytope. We apply (5.1) when

$$
A=B(\varepsilon)=P \backslash \bigcup_{T} S(T, \varepsilon)
$$

where $\varepsilon=(\log n)^{-1}$. We use the method of [BáLa]. Changing $n$ to $n+1$ and applying (3.5) we get

$$
\begin{aligned}
& (n+1) \int_{B(\varepsilon)} \operatorname{Prob}\left(x \notin P_{n}\right) d x \\
& \leq(n+1) \int_{B(\varepsilon)} 2 \sum_{i=0}^{d-1}\left(\begin{array}{c}
n \\
i
\end{array}\right)\left(\frac{u(x)}{2}\right)^{i}\left(1-\frac{u(x)}{2}\right)^{n-i} d x \\
& =(n+1) \sum_{\lambda=1}^{n} \int_{B(\varepsilon)} 2 \sum_{i=0}^{d-1}\left(\begin{array}{c}
n \\
i
\end{array}\right)\left(\frac{u(x)}{2}\right)^{i}\left(1-\frac{u(x)}{2}\right)^{n-i} d x \\
& \leq 2(n+1) \sum_{\lambda=1}^{n} \sum_{i=0}^{\frac{\lambda-1}{n} \leq u(x) \leq \frac{\lambda}{n}}\left(\begin{array}{c}
n \\
i
\end{array}\right)\left(\frac{\lambda}{2 n}\right)^{i}\left(1-\frac{\lambda-1}{2 n}\right)^{n-i} \operatorname{vol}\left\{x \in B(\varepsilon): u(x) \leq \frac{\lambda}{n}\right\} .
\end{aligned}
$$

Here $\left(\begin{array}{c}n \\ i\end{array}\right)\left(\frac{\lambda}{2 n}\right)^{i} \leq \frac{\lambda^{i}}{2^{i} i !},\left(1-\frac{\lambda-1}{2 n}\right)^{-i} \leq 2^{i}$, and $\left(1-\frac{\lambda-1}{2 n}\right)^{n} \leq e^{-(\lambda-1) / 2}$ yield

$$
\sum_{i=0}^{d-1}\left(\begin{array}{l}
n \\
i
\end{array}\right)\left(\frac{\lambda}{2 n}\right)^{i}\left(1-\frac{\lambda-1}{2 n}\right)^{n-i} \leq \mathrm{const}(d) \lambda^{d-1} e^{-\lambda / 2} .
$$

Moreover, $\operatorname{vol}\left\{x \in B(\varepsilon): u(x) \leq \frac{\lambda}{n}\right\} \leq 1$. Set $\lambda_{0}=\lfloor 4 \log n\rfloor$. Then 


$$
\begin{aligned}
& 2(n+1) \sum_{\lambda=\lambda_{0}+1}^{n} \sum_{i=0}^{d-1}\left(\begin{array}{c}
n \\
i
\end{array}\right)\left(\frac{\lambda}{2 n}\right)^{i}\left(1-\frac{\lambda-1}{2 n}\right)^{n-i} \operatorname{vol}\left\{x \in B(\varepsilon): u(x) \leq \frac{\lambda}{n}\right\} \\
& \leq \operatorname{const}(d) n \sum_{\lambda=\lambda_{0}+1}^{n} \lambda^{d-1} e^{-\lambda / 2} \\
& \leq \operatorname{const}(d) n e^{-\lambda_{0} / 4} \sum_{\lambda=1}^{\infty} \lambda^{d-1} e^{-\lambda / 4} \\
& \leq \operatorname{const}(d) .
\end{aligned}
$$

We know from Theorem 6 or rather from (4.4) that

$$
\operatorname{vol}\left\{x \in B(\varepsilon): u(x) \leq \frac{\lambda}{n}\right\} \leq \operatorname{const}(P) \frac{\lambda}{n} \log ^{d-2} \frac{n}{\lambda} \log \frac{1}{\varepsilon},
$$

since $\varepsilon=(\log n)^{-1}$ satisfies $\varepsilon^{d} \geq \operatorname{const}(P) \frac{\lambda}{n}$ when $\lambda \leq \lambda_{0}$. So we have

$$
\begin{aligned}
& 2(n+1) \sum_{\lambda=1}^{\lambda_{0}} \sum_{i=0}^{d-1}\left(\begin{array}{l}
n \\
i
\end{array}\right)\left(\frac{\lambda}{2 n}\right)^{i}\left(1-\frac{\lambda-1}{2 n}\right)^{n-i} \operatorname{vol}\left\{x \in B(\varepsilon): u(x) \leq \frac{\lambda}{n}\right\} \\
& \leq \operatorname{const}(d) n \sum_{\lambda=1}^{\lambda_{0}} \lambda^{d-1} e^{-\lambda / 2} \operatorname{const}(P) \frac{\lambda}{n} \log { }^{d-2} \frac{n}{\lambda} \log \log n \\
& \leq \operatorname{const}(P) \sum_{\lambda=1}^{\lambda_{0}} \lambda^{d} e^{-\lambda / 2} \log ^{d-2} n \log \log n \\
& \leq \operatorname{const}(P) \log ^{d-2} n \log \log n .
\end{aligned}
$$

This proof will serve as a model for some proofs to come. In particular, estimations analogous to (5.2), (5.3), (5.4), and (5.5) will frequently be used with reference to this section and without elaboration.

\section{Proof of Theorem 4}

Again let $X_{n}=\left\{x_{1}, \ldots, x_{n}\right\}$ be the set of the $n$ random points in $P$. For $i=$ $0,1, \ldots, d-1$ define

$$
\begin{aligned}
E(i, n)= & E\left[\operatorname{vert} \operatorname{conv}\left(X_{n} \cap P\left(\bar{\phi}_{i}\right)\right) \text { in } P\left(\bar{\varphi}_{i}\right)\right] \\
& -E\left[\operatorname{vert} \operatorname{conv}\left(X_{n} \cap P\left(\bar{\phi}_{i-1}\right)\right) \text { in } P\left(\bar{\varphi}_{i}\right)\right] .
\end{aligned}
$$

Here $P\left(\ddot{\phi}_{i}\right)$ and $P\left(\tilde{\varphi}_{i}\right)$ are defined in (2.6), cf. (2.7) as well. We set

$$
\varphi_{i}=(\log n)^{-1}, \phi_{i}=\operatorname{const}(P) \quad(i=0,1, \ldots, d-1)
$$

where $\phi_{i}$ is chosen so small that the set $\left\{z \in P: 0<\tau_{i}(z)<2 \phi_{i}\right\}$ does not contain any vertex of $P$. We claim that

$$
0 \leq E(i, n) \leq \operatorname{const}(P) \log ^{d-2} n \log \log n .
$$


This will prove the theorem in the following way:

$$
\begin{aligned}
E\left(\text { vert } P_{n}\right)= & \sum_{F_{0}} E\left[\operatorname{vert} P_{n} \text { in } P\left(\varphi_{0} ; F_{0}\right)\right] \\
& +O\left(\log ^{d-2} n \log \log n\right) \\
= & \sum_{F_{0}} E\left[\operatorname{vert} \operatorname{conv}\left(X_{n} \cap P\left(\phi_{0} ; F_{0}\right)\right) \text { in } P\left(\varphi_{0} ; F_{0}\right)\right] \\
& +O\left(\log ^{d-2} n \log \log n\right) \\
= & \sum_{F_{0}, F_{1}} E\left[\operatorname{vert} \operatorname{conv}\left(X_{n} \cap P\left(\phi_{0} ; F_{0}\right)\right) \text { in } P\left(\varphi_{0}, \varphi_{1} ; F_{0}, F_{1}\right)\right] \\
& +O\left(\log ^{d-2} n \log \log n\right) \\
= & \sum_{F_{0}, F_{1}} E\left[\operatorname{vert} \operatorname{conv}\left(X_{n} \cap P\left(\phi_{0}, \phi_{1} ; F_{0}, F_{1}\right)\right) \text { in } P\left(\varphi_{0}, \varphi_{1} ; F_{0}, F_{1}\right)\right] \\
& +O\left(\log ^{d-2} n \log \log n\right) \\
= & \ldots \\
= & \sum_{T} E\left[\operatorname{vert} \operatorname{conv}\left(X_{n} \cap P\left(\phi_{0}, \ldots, \phi_{d-1} ; T\right)\right) \text { in } P\left(\varphi_{0}, \ldots, \varphi_{d-1} ; T\right)\right] \\
& +O\left(\log ^{d-2} n \log \log n\right)
\end{aligned}
$$

where the equalities follow from Theorem 3 and (6.2), alternatively. The terms in the last sum are independent of $P$, they depend only on $\varphi_{0}, \ldots, \varphi_{d-1}$ and $\phi_{0}, \ldots, \phi_{d-1}$. This means that they are the same for every tower of every polytope once these numbers are equal. For a simple polytope Affentranger and Wieacker determined

$$
E\left(\text { vert } P_{n}\right)=\frac{d \text { vert } P}{(d+1)^{d-1}} \log ^{d-1} n+O\left(\log ^{d-2} n\right) .
$$

Since $T(P)=d$ ! vert $P$ for a simple polytope, we get from (6.3) that the expected number of vertices of $P_{n}$ lying in $S\left(T,(\log n)^{-1}\right)$ is

$$
\frac{1}{(d+1)^{d-1}(d-1) !} \log ^{d-1} n+O\left(\log ^{d-2} n \log \log n\right) .
$$

But then $E\left[\right.$ vert $P_{n}$ in $\left.S\left(T,(\log n)^{-1}\right)\right]$ is this very number for every tower $T$ of every polytope, simple or otherwise.

Set $q=\operatorname{vol} P\left(\bar{\phi}_{i-1}\right)$. Choosing the random $n$-set $X_{n}$ from $P$ is the same as the following two-step procedure. First choose $m \in\{0,1, \ldots, n\}$ with probability $\left(\begin{array}{l}n \\ m\end{array}\right) q^{m}(1-q)^{n-m}$, then choose $m$ points $y_{1}, \ldots, y_{m}$ from $P\left(\bar{\phi}_{i-1}\right)$ randomly, independently and uniformly, and choose $n-m$ points from $P \backslash P\left(\bar{\phi}_{i-1}\right)$ randomly, independently and uniformly. Correspondingly,

$$
\begin{aligned}
E(i, n)= & \sum_{m=0}^{n}\left(\begin{array}{c}
n \\
m
\end{array}\right) q^{m}(1-q)^{n-m} \\
& \left\{E\left[\operatorname{vert} \operatorname{conv}\left(X_{n} \cap P\left(\bar{\phi}_{i}\right)\right) \text { in } P\left(\bar{\varphi}_{i}\right) \mid \operatorname{card}\left(X_{n} \cap P\left(\bar{\phi}_{i-1}\right)\right)=m\right]\right. \\
& \left.-E\left[\operatorname{vert} \operatorname{conv}\left(X_{n} \cap P\left(\bar{\phi}_{i-1}\right)\right) \text { in } P\left(\bar{\varphi}_{i}\right) \mid \operatorname{card}\left(X_{n} \cap P\left(\bar{\phi}_{i-1}\right)\right)=m\right]\right\}
\end{aligned}
$$




$$
\begin{aligned}
= & \sum_{m=0}^{n}\left(\begin{array}{l}
n \\
m
\end{array}\right) q^{m}(1-q)^{n-m} \\
& \left\{E\left[\operatorname{vert} \operatorname{conv}\left(Y_{m} \cap P\left(\bar{\phi}_{i}\right)\right) \text { in } P\left(\bar{\varphi}_{i}\right)\right]\right. \\
& \left.-E\left[\operatorname{vert} \operatorname{conv}\left(Y_{m} \cap P\left(\bar{\phi}_{i-1}\right)\right) \text { in } P\left(\bar{\varphi}_{i}\right)\right]\right\}
\end{aligned}
$$

with $Y_{m}=\left\{y_{1}, \ldots, y_{m}\right\}$. Here conv $\left(Y_{m} \cap P\left(\bar{\phi}_{i-1}\right)\right)=P\left(\bar{\phi}_{i-1}\right)_{m}$ since $Y_{m} \subset P\left(\bar{\phi}_{i-1}\right)$, but we cannot use the same notation for conv $\left(Y_{m} \cap P\left(\bar{\phi}_{i}\right)\right)$. So we better leave them as they are. We continue (6.4) using (5.1)

$$
\begin{aligned}
E(i, n)= & \sum_{m=0}^{n}\left(\begin{array}{c}
n \\
m
\end{array}\right) q^{m}(1-q)^{n-m} \\
& m \int_{x \in P\left(\bar{\varphi}_{i}\right)} \operatorname{Prob}\left[x \notin \operatorname{conv}\left(Y_{m-1} \cap P\left(\tilde{\phi}_{i}\right)\right)\right. \\
& \text { and } \left.x \in \operatorname{conv}\left(Y_{m-1} \cap P\left(\bar{\phi}_{i-1}\right)\right)\right] d x .
\end{aligned}
$$

So we see that $E(i, n) \geq 0$. We claim now that for $m \geq d+2$

$$
\begin{aligned}
E_{0}:= & m \int_{x \in P\left(\bar{\varphi}_{i}\right)} \operatorname{Prob}\left[x \notin \operatorname{conv}\left(Y_{m-1} \cap P\left(\bar{\phi}_{i}\right)\right)\right. \\
& \text { and } \left.x \in \operatorname{conv}\left(Y_{m-1} \cap P\left(\bar{\phi}_{i-1}\right)\right)\right] d x \\
\leq & \operatorname{const}(P) \log ^{d-2} m \log \log m .
\end{aligned}
$$

( $E_{0}=0$ clearly for $m \leq d+1$.) This will prove (6.2), since using (6.6) in (6.5) gives

$$
\begin{aligned}
E(i, n) & \leq \sum_{m=d+2}^{n}\left(\begin{array}{c}
n \\
m
\end{array}\right) q^{m}(1-q)^{n-m} \operatorname{const}(P) \log ^{d-2} m \log \log m \\
& \leq \operatorname{const}(P) \log ^{d-2} n \log \log n \sum_{m=d+2}^{n}\left(\begin{array}{c}
n \\
m
\end{array}\right) q^{m}(1-q)^{n-m} \\
& \leq \operatorname{const}(P) \log ^{d-2} n \log \log n .
\end{aligned}
$$

As we prove (6.6) we now introduce the notation $K=P\left(\bar{\phi}_{i-1}\right)$, and we assume that vol $K=\operatorname{vol} P\left(\bar{\phi}_{i-1}\right)=1$ since in (6.6) this does not matter. Let us write further

$$
\begin{aligned}
& K\left(\tau_{i} \leq \phi_{i}\right):=P\left(\tilde{\phi}_{i}\right), \\
& K\left(\tau_{i} \geq \phi_{i}\right):=\left\{z \in K: \tau_{i}(z) \geq \phi_{i}\right\}, \\
& K\left(\tau_{i} \geq \varphi_{i}\right):=\left\{z \in K: \tau_{i}(z) \geq \varphi_{i}\right\},
\end{aligned}
$$

but $P\left(\tilde{\varphi}_{i}\right)=P\left(\varphi_{0}, \ldots, \varphi_{i}\right)$ as earlier. For the estimation (6.6) we need the simple but important

Proposition 1. Assume $x, y_{1}, \ldots, y_{m-1}$ are in general position in $K$. Set $Y_{m-1}=$ $\left\{y_{1}, \ldots, y_{m-1}\right\}$ and assume, further, that

$$
x \in P\left(\bar{\varphi}_{i}\right), x \in \operatorname{conv} Y_{m-1}, x \notin \operatorname{conv}\left(Y_{m-1} \cap K\left(\tau_{i} \leq \phi_{i}\right)\right) .
$$

Then there is a $y_{k} \in Y_{m-1} \cap K\left(\tau_{i} \geq \phi_{i}\right)$ such that 


$$
\operatorname{ray}\left(x, y_{k}\right) \cap \operatorname{conv}\left[\left(Y_{m-1} \backslash\left\{y_{k}\right\}\right) \cap K\left(\tau_{i} \geq \varphi_{i}\right)\right]=\emptyset
$$

and

$$
\operatorname{ray}\left(x, y_{k}\right) \cap \operatorname{conv}\left[\left(Y_{m-1} \backslash\left\{y_{k}\right\}\right) \cap K\left(\tau_{i} \leq \phi_{i}\right)\right]=\emptyset
$$

Proof. Identify $x$ with the origin for this proof. Then the conditions imply that

$$
\begin{aligned}
& C_{1}:=\text { cone } Y_{m-1}=\mathbb{R}^{d}, \\
& C_{2}:=\operatorname{cone}\left(Y_{m-1} \cap K\left(\tau_{i} \leq \phi_{i}\right)\right) \neq \mathbb{R}^{d}, \\
& C_{3}:=\operatorname{cone}\left(Y_{m-1} \cap K\left(\tau_{i} \geq \varphi_{i}\right)\right) \neq \mathbb{R}^{d} .
\end{aligned}
$$

As the sum of the last two cones is $C_{1}, C_{3}$ must have an extreme ray, defined by some $y_{k} \in Y_{m-1} \cap K\left(\tau_{i} \geq \varphi_{i}\right)$ that is not in $C_{2}$. Then $y_{k} \notin K\left(\tau_{i} \leq \phi_{i}\right)$ as well, and $\operatorname{ray}\left(x, y_{k}\right)$ has the claimed properties.

We rewrite (6.6) using the new notation and Proposition 1.

$$
\begin{aligned}
& E_{0}=m \int_{P\left(\bar{\varphi}_{i}\right)} \operatorname{Prob}\left[x \notin \operatorname{conv}\left(Y_{m-1} \cap K\left(\tau_{i} \leq \phi_{i}\right)\right) \text { and } x \in \operatorname{conv}\left(Y_{m-1} \cap K\right)\right] d x \\
& \leq m \int_{P\left(\bar{\varphi}_{i}\right)} \operatorname{Prob}\left[\exists y_{k} \in Y_{m-1} \cap K\left(\tau_{i} \geq \phi_{i}\right) \text { such that (6.7) and (6.8) hold }\right] d x \\
& \leq m \int_{P\left(\bar{\varphi}_{i}\right)} \sum_{k=1}^{m-1} \operatorname{Prob}\left[y_{k} \in K\left(\tau_{i} \geq \phi_{i}\right) \text { and }(6.7) \text { and }(6.8) \text { hold }\right] d x \\
& \leq m \int_{x \in P\left(\bar{\varphi}_{i}\right)}(m-1) \int_{y \in K\left(r_{i} \geq \phi_{i}\right)} \operatorname{Prob}\left[\operatorname{ray}(x, y) \cap \operatorname{conv}\left(Y_{m-2} \cap K\left(\tau_{i} \geq \varphi_{i}\right)\right)=\emptyset\right. \\
&\text { and } \left.\operatorname{ray}(x, y) \cap \operatorname{conv}\left(Y_{m-2} \cap K\left(\tau_{i} \leq \phi_{i}\right)\right)=\emptyset\right] d y d x .
\end{aligned}
$$

Now change $m$ to $m+2$ and define the events

$$
\begin{aligned}
& G 1: \operatorname{ray}(x, y) \cap \operatorname{conv}\left(Y_{m} \cap K\left(\tau_{i} \geq \varphi_{i}\right)\right)=\emptyset, \\
& G 2: \operatorname{ray}(x, y) \cap \operatorname{conv}\left(Y_{m} \cap K\left(\tau_{i} \leq \phi_{i}\right)\right)=\emptyset .
\end{aligned}
$$

Thus, in order to prove (6.6) it will be enough to show that

$$
m^{2} \iint_{(x, y) \in K^{(i)}} \operatorname{Prob}(G 1 \text { and } G 2) d y d x \leq \operatorname{const}(P) \log ^{d-2} m \log \log m
$$

where $K^{(i)}=P\left(\tilde{\varphi}_{i}\right) \times K\left(\tau_{i} \geq \phi_{i}\right)$.

Let $z$ be the point where the function

$$
u=u_{K}\left(=u_{P\left(\bar{\phi}_{i-1}\right)}\right)
$$

takes its maximum value on aff $(x, y)$. It is known that $z$ is unique (cf. Section 3), but we will not need this. We split $K^{(i)}$, the domain of the integration in (6.9), into three parts: 


$$
\begin{aligned}
& K_{1}^{(i)}=\left\{(x, y) \in K^{(i)}: \tau_{i}(z) \geq 2 \varphi_{i}\right\}, \\
& K_{2}^{(i)}=\left\{(x, y) \in K^{(i)}: \tau_{i}(x) \leq \tau_{i}(z) \leq 2 \varphi_{i}\right\}, \\
& K_{3}^{(i)}=\left\{(x, y) \in K^{(i)}: \tau_{i}(z) \leq \tau_{i}(x)\right\} .
\end{aligned}
$$

We will estimate the integral (6.9) separately for the three parts.

Case 1: $\tau_{i}(z) \geq 2 \varphi_{i}$. Set $\bar{u}=u_{K\left(r_{i} \geq \varphi_{i}\right)}, \bar{q}=\operatorname{vol} K\left(\tau_{i} \geq \varphi_{i}\right)$, and recall (3.5).

$\operatorname{Prob}(G 1$ and $G 2) \leq \operatorname{Prob}(G 1)$

$\leq \operatorname{Prob}\left[z \notin \operatorname{conv}\left(Y_{m} \cap K\left(\tau_{i} \geq \varphi_{i}\right)\right)\right]$

$=\sum_{\mu=0}^{m}\left(\begin{array}{l}m \\ \mu\end{array}\right) \bar{q}^{\mu}(1-\bar{q})^{m-\mu}$

$\operatorname{Prob}\left[z \notin \operatorname{conv}\left(Y_{m} \cap K\left(\tau_{i} \geq \varphi_{i}\right)\right) \mid \operatorname{card}\left(Y_{m} \cap K\left(\tau_{i} \geq \varphi_{i}\right)\right)=\mu\right]$

$=\sum_{\mu=0}^{m}\left(\begin{array}{l}m \\ \mu\end{array}\right) \bar{q}^{\mu}(1-\bar{q})^{m-\mu} \operatorname{Prob}\left(z \notin K\left(\tau_{i} \geq \varphi_{i}\right)_{\mu}\right)$

$\leq \sum_{\mu=0}^{m}\left(\begin{array}{c}m \\ \mu\end{array}\right) \bar{q}^{\mu}(1-\bar{q})^{m-\mu} 2 \sum_{j=0}^{d-1}\left(\begin{array}{c}\mu \\ j\end{array}\right)\left(\frac{\bar{u}(z)}{2 \bar{q}}\right)^{j}\left(1-\frac{\bar{u}(z)}{2 \bar{q}}\right)^{\mu-j}$

$=2 \sum_{j=0}^{d-1}\left(\begin{array}{c}m \\ j\end{array}\right) \bar{q}^{j}\left(\frac{\bar{u}(z)}{2 \bar{q}}\right)^{j} \sum_{\mu=j}^{m}\left(\begin{array}{c}m-j \\ m-\mu\end{array}\right)(1-\bar{q})^{m-\mu}\left[\bar{q}\left(1-\frac{\bar{u}(z)}{2 \bar{q}}\right)\right]^{\mu-j}$

$=2 \sum_{j=0}^{d-1}\left(\begin{array}{c}m \\ j\end{array}\right)\left(\frac{\bar{u}(z)}{2}\right)^{j} \sum_{\mu=0}^{m-j}\left(\begin{array}{c}m-j \\ m-j-\mu\end{array}\right)(1-\bar{q})^{m-j-\mu}\left(\bar{q}-\frac{\bar{u}(z)}{2}\right)^{\mu}$

$=2 \sum_{j=0}^{d-1}\left(\begin{array}{c}m \\ j\end{array}\right)\left(\frac{\bar{u}(z)}{2}\right)^{j}\left(1-\frac{\bar{u}(z)}{2}\right)^{m-j}$.

Then

$$
\begin{aligned}
E_{1} & :=m^{2} \iint_{K_{1}^{(i)}} \operatorname{Prob}(G 1 \text { and } G 2) d y d x \\
& \leq m^{2} \iint_{K_{1}^{(i)}} 2 \sum_{j=0}^{d-1}\left(\begin{array}{c}
m \\
j
\end{array}\right)\left(\frac{\bar{u}(z)}{2}\right)^{j}\left(1-\frac{\bar{u}(z)}{2}\right)^{m-j} d y d x \\
& =2 m^{2} \sum_{\lambda=1}^{m} \iint_{K_{1}^{(i)}} \sum_{j=0}^{d-1}\left(\begin{array}{c}
m \\
j
\end{array}\right)\left(\frac{\bar{u}(z)}{2}\right)^{j}\left(1-\frac{\bar{u}(z)}{2}\right)^{m-j} d y d x \\
& \leq \operatorname{const}(d) m^{2} \sum_{\lambda=1}^{m} \lambda^{d-1} e^{-\lambda / 2} \operatorname{meas}\left\{(x, y) \in K_{1}^{(i)}: \bar{u}(z) \leq \frac{\lambda}{m}\right\}
\end{aligned}
$$

where the last inequality follows in the same way as (5.2) and (5.3). This time we set $\lambda_{0}=\lfloor 8 \log m\rfloor$ and write 


$$
\begin{aligned}
E_{1} \leq & \operatorname{const}(d) m^{2}\left[\sum_{\lambda=1}^{\lambda_{0}} \lambda^{d-1} e^{-\lambda / 2} \operatorname{meas}\left\{(x, y) \in K_{1}^{(i)}: \bar{u}(z) \leq \frac{\lambda}{m}\right\}\right. \\
& \left.+\sum_{\lambda=\lambda_{0}+1}^{m} \lambda^{d-1} e^{-\lambda / 2}\right] .
\end{aligned}
$$

The second sum is less than const $(d) m^{-2}$; cf, (5.4). For the first sum we need

Proposition 2. $u(z) \leq \frac{2 d !}{(d-i) !} \bar{u}(z)$ if $\tau_{i}(z) \geq 2 \varphi_{i}$.

Proof. We use induction. For $i=0$ the statement is

$$
u_{P}(z) \leq 2 u_{P\left(\tau_{0} \geq \varphi_{0}\right)}(z)
$$

provided $\tau_{0}(z) \geq 2 \varphi_{0}$. Observe that $M_{P\left(\tau_{0} \geq \varphi_{0}\right)}(z)=M_{P}(z) \cap H_{0}\left(\varphi_{0}, 2 \tau_{0}(z)-\varphi_{0}\right)$ where $H_{0}\left(t_{1}, t_{2}\right)$ stands for the slab between $H_{0}\left(t_{1}\right)$ and $H_{0}\left(t_{2}\right)$. So by (3.2)

$$
\begin{aligned}
\frac{\tilde{u}(z)}{u(z)} & =\frac{2 \int_{0}^{\tau_{0}(z)} \operatorname{vol}_{d-1}\left[M_{P\left(\tau_{0} \geq \varphi_{0}\right)}(z) \cap H_{0}(t)\right] d t}{2 \int_{0}^{\tau_{0}(z)} \operatorname{vol}_{d-1}\left[M_{P}(z) \cap H_{0}(t)\right] d t} \\
& =\frac{\int_{\varphi_{0}}^{\tau_{0}(z)} \operatorname{vol}_{d-1}\left[M_{P}(z) \cap H_{0}(t)\right] d t}{\int_{0}^{\tau_{0}(z)} \operatorname{vol}_{d-1}\left[M_{P}(z) \cap H_{0}(t)\right] d t} \geq \frac{1}{2}
\end{aligned}
$$

since $\tau_{0}(z) \geq 2 \varphi_{0}$ and the integrand is a monotone function.

When $i \geq 1, t_{0}:=\max \left\{0,2 \tau_{0}(z)-\phi_{0}\right\}$ is the smallest $t$ such that

$$
M_{P\left(\phi_{0}, \ldots, \phi_{i-1}\right)}(z) \cap H_{0}(t) \text { and } M_{P\left(\phi_{0}, \ldots, \phi_{i-1}, \tau_{i} \geq \varphi_{i}\right)}(z) \cap H_{0}(t)
$$

are nonempty. Therefore (3.3), (3.4), and the induction hypothesis (also $c f .(2.8)$ ) imply

$$
\begin{aligned}
\frac{\bar{u}(z)}{u(z)} & =\frac{\operatorname{vol} M_{P\left(\phi_{0}, \ldots, \phi_{i-1}, \tau_{i} \geq \varphi_{i}\right)}(z)}{\operatorname{vol} M_{P\left(\phi_{0}, \ldots, \phi_{i-1}\right)}(z)} \\
& =\frac{2 \int_{t_{0}}^{\tau_{0}(z)} \operatorname{vol}_{d-1}\left[M_{P\left(\phi_{0}, \ldots, \phi_{i-1}, \tau_{i} \geq \varphi_{i}\right)}(z) \cap H_{0}(t)\right] d t}{2 \int_{t_{0}(z)}^{\tau_{0}(z)} \operatorname{vol}_{d-1}\left[M_{P\left(\phi_{0}, \ldots, \phi_{i-1}\right)}(z) \cap H_{0}(t)\right] d t} \\
& \geq \frac{\frac{\tau_{0}(z)-t_{0}}{d} \operatorname{vol}_{d-1}\left[M_{P\left(\phi_{0}, \ldots, \phi_{i-1}, \tau_{i} \geq \varphi_{i}\right)}(z) \cap H_{0}\left(\tau_{0}(z)\right)\right]}{\left(\tau_{0}(z)-t_{0}\right) \operatorname{vol}_{d-1}\left[M_{P\left(\phi_{0}, \ldots, \phi_{i-1}\right)}(z) \cap H_{0}\left(\tau_{0}(z)\right)\right]} \\
& =\frac{1 u_{Q\left(\tau_{0}(z)\right)\left(\phi_{1}, \ldots, \phi_{i-1}, \tau_{i} \geq \varphi_{i}\right)}(z)}{d} \geq \frac{1}{d} \frac{(d-i) !}{(d-1) !} \frac{1}{u_{Q\left(\tau_{0}(z)\right)\left(\phi_{1}, \ldots, \phi_{i-1}\right)}(z)} .
\end{aligned}
$$




$$
\begin{aligned}
& \sum_{\lambda=1}^{\lambda_{0}} \lambda^{d-1} e^{-\lambda / 2} \text { meas }\left\{(x, y) \in K_{1}^{(i)}: \bar{u}(z) \leq \frac{\lambda}{m}\right\} \\
& \leq \sum_{\lambda=1}^{\lambda_{0}} \lambda^{d-1} e^{-\lambda / 2} \operatorname{meas}\left\{(x, y) \in K_{1}^{(i)}: u(z) \leq 2 d ! \frac{\lambda}{m}\right\} \\
& \leq \sum_{\lambda=1}^{\lambda_{0}} \lambda^{d-1} e^{-\lambda / 2} \operatorname{const}(P)\left(\frac{2 d ! \lambda}{m}\right)^{2} \log ^{d-2} \frac{m}{2 d ! \lambda} \log \log \frac{m}{2 d ! \lambda} \\
& \leq \operatorname{const}(P) m^{-2} \log ^{d-2} m \log \log m .
\end{aligned}
$$

This proves that

$$
E_{1} \leq \operatorname{const}(P) \log ^{d-2} m \log \log m
$$

Case 2: $\tau_{i}(x) \leq \tau_{i}(z) \leq 2 \varphi_{i}$. This time we set $\tilde{u}=u_{K\left(\tau_{i} \leq \phi_{i}\right)}$ and $\tilde{q}=\operatorname{vol} K\left(\tau_{i} \leq \phi_{i}\right)$. In a similar way as in Case 1 we see that

$$
\begin{aligned}
& \operatorname{Prob}(G 1 \text { and } G 2) \leq \operatorname{Prob}(G 2) \\
& \leq \operatorname{Prob}\left[z \notin \operatorname{conv}\left(Y_{m} \cap K\left(\tau_{i} \leq \phi_{i}\right)\right)\right] \\
& \leq 2 \sum_{j=0}^{d-1}\left(\begin{array}{c}
m \\
j
\end{array}\right)\left(\frac{\tilde{u}(z)}{2}\right)^{j}\left(1-\frac{\tilde{u}(z)}{2}\right)^{m-j}
\end{aligned}
$$

Correspondingly,

$$
\begin{aligned}
E_{2}:= & m^{2} \iint_{K_{2}^{(i)}} \operatorname{Prob}(G 1 \text { and } G 2) d y d x \\
& \leq m^{2} \iint_{K_{2}^{(i)}} 2 \sum_{j=0}^{d-1}\left(\begin{array}{c}
m \\
j
\end{array}\right)\left(\frac{\tilde{u}(z)}{2}\right)^{j}\left(1-\frac{\tilde{u}(z)}{2}\right)^{m-j} \\
\leq & \operatorname{const}(d) m^{2} \sum_{\lambda=1}^{m} \lambda^{d-1} e^{-\lambda / 2} \operatorname{meas}\left\{(x, y) \in K_{2}^{(i)}: \tilde{u}(z) \leq \frac{\lambda}{m}\right\} \\
\leq & \operatorname{const}(d) m^{2}\left[\sum_{\lambda=1}^{\lambda_{0}} \lambda^{d-1} e^{-\lambda / 2} \operatorname{meas}\left\{(x, y) \in K_{2}^{(i)}: \tilde{u}(z) \leq \frac{\lambda}{m}\right\}\right. \\
& \left.+\sum_{\lambda=\lambda_{0}+1}^{m} \lambda^{d-1} e^{-\lambda / 2}\right]
\end{aligned}
$$

where $\lambda_{0}=\lfloor 8 \log m\rfloor$, again. Here we need

Proposition 3. $u(z) \leq \frac{d !}{(d-i) !} \tilde{u}(z)$ if $\tau_{i}(z) \leq \frac{\phi_{i}}{2}$.

Proof. By induction again. The case $i=0$ is very simple, since $u_{P}(z)=u_{P\left(\phi_{0}\right)}(z)$ if $\tau_{0}(z) \leq \frac{\phi_{0}}{2}$. When $i \geq 1$, the same reasoning as in the proof of Proposition 2 gives 


$$
\begin{aligned}
\frac{\tilde{u}(z)}{u(z)} & =\frac{\operatorname{vol} M_{P\left(\phi_{0}, \ldots, \phi_{i}\right)}(z)}{\operatorname{vol} M_{P\left(\phi_{0}, \ldots, \phi_{i-1}\right)}(z)} \\
& =\frac{2 \int_{t_{0}}^{\tau_{0}(z)} \operatorname{vol}_{d-1}\left[M_{P\left(\phi_{0}, \ldots, \phi_{i}\right)}(z) \cap H_{0}(t)\right] d t}{2 \int_{t_{0}}^{\tau_{0}(z)} \operatorname{vol}_{d-1}\left[M_{P\left(\phi_{0}, \ldots, \phi_{i-1}\right)}(z) \cap H_{0}(t)\right] d t} \\
& \geq \frac{\frac{1}{d}\left(\tau_{0}(z)-t_{0}\right) \operatorname{vol}_{d-1}\left[M_{P\left(\phi_{0}, \ldots, \phi_{i}\right)}(z) \cap H_{0}\left(\tau_{0}(z)\right)\right]}{\left(\tau_{0}(z)-t_{0}\right) \operatorname{vol}_{d-1}\left[M_{P\left(\phi_{0}, \ldots, \phi_{i-1}\right)}(z) \cap H_{0}\left(\tau_{0}(z)\right)\right]} \\
& \geq \frac{1}{d} \frac{u_{Q\left(\tau_{0}(z)\right)\left(\phi_{1}, \ldots, \phi_{i}\right)}(z)}{u_{Q\left(\tau_{0}(z)\right)\left(\phi_{1}, \ldots, \phi_{i-1}\right)}(z)} \geq \frac{1}{d} \frac{(d-i) !}{(d-1) !}
\end{aligned}
$$

Observing (6.1) we see in the same way as in Case 1 that

$$
E_{2} \leq \mathrm{const}(P) \log ^{d-2} m \log \log m
$$

Case 3: $\tau_{i}(z) \leq \tau_{i}(x)$. Of course, $\tau_{i}(x) \leq \varphi_{i}<\phi_{i} \leq \tau_{i}(y)$. Macbeath proved that the set $\{x \in K: u(x) \geq \varepsilon\}$ is convex (recall Section 2). This implies that $u$ is maximal on ray $(x, y)$ at $x$. Similarly as in Case 2 - but with $x$ instead of $z$-we get

$$
\begin{aligned}
E_{3}:= & m^{2} \iint_{K_{3}^{(i)}} \operatorname{Prob}(G 1 \text { and } G 2) d y d x \\
\leq & m^{2} \iint_{K_{3}^{(i)}} \operatorname{Prob}\left[x \notin \operatorname{conv}\left(Y_{m} \cap K\left(\tau_{i} \leq \phi_{i}\right)\right)\right] d y d x \\
\leq & \operatorname{const}(d) m^{2}\left[\sum_{\lambda=0}^{\lambda_{0}} \lambda^{d-1} e^{-\lambda / 2} \operatorname{meas}\left\{(x, y) \in K_{3}^{(i)}: \tilde{u}(x) \leq \frac{\lambda}{m}\right\}\right. \\
& \left.+\sum_{\lambda=\lambda_{0}+1}^{m} \lambda^{d-1} e^{-\lambda / 2}\right]
\end{aligned}
$$

with $\lambda_{0}=\lfloor 8 \log m\rfloor$. Again $u(x) \leq d ! u(x)$ by Proposition 3. Lemma 1 shows that $y \in M_{K}(z, \theta)$ with $\theta=(3 d)^{d+2}$. As $x$ lies on the segment connecting $z$ and $y$ we have $y \in M_{K}(x, \theta)$. Hence

$$
\begin{aligned}
\operatorname{meas} & \left\{(x, y) \in K_{3}^{(i)}: \tilde{u}(x) \leq \frac{\lambda}{m}\right\} \\
& \leq \operatorname{meas}\left\{(x, y) \in K_{3}^{(i)}: u(x) \leq d ! \frac{\lambda}{m}\right\} \\
& \leq \operatorname{meas}\left\{(x, y) \in P\left(\bar{\varphi}_{i}\right) \times K\left(\tau_{i} \geq \phi_{i}\right): u(x) \leq d ! \frac{\lambda}{m}, y \in M_{K}(x, \theta)\right\} \\
& =\int_{\substack{x \in P\left(\phi_{i}\right) \\
u(x) \leq d i}} \operatorname{vol}\left\{y \in K\left(\tau_{i} \geq \phi_{i}\right): y \in M_{K}(x, \theta)\right\} d x
\end{aligned}
$$


Estimating the integrand by Lemma 2 and observing (6.1) we further see that

$$
\begin{aligned}
\operatorname{meas} & \left\{(x, y) \in K_{3}^{(i)}: \tilde{u}(x) \leq \frac{\lambda}{m}\right\} \\
& \leq \int_{\substack{x \in P\left(\bar{\varphi}_{i}\right) \\
u(x) \leq d ! \frac{\lambda}{m}}} \operatorname{const}(P) \tau_{i}(x) u(x) d x \\
& \leq \operatorname{const}(P)(\log m)^{-1} \frac{d ! \lambda}{m} \int_{\substack{x \in P\left(\bar{\varphi}_{i}\right) \\
u(x) \leq d ! \frac{\lambda}{m}}} 1 d x .
\end{aligned}
$$

By Theorem 6

$$
\operatorname{vol}\left\{x \in P\left(\bar{\varphi}_{i}\right): u(x) \leq d ! \frac{\lambda}{m}\right\} \leq \operatorname{const}(d) \frac{d ! \lambda}{m} \log ^{d-1} \frac{d ! \lambda}{m},
$$

and therefore

$$
\begin{gathered}
\operatorname{meas}\left\{(x, y) \in K_{3}^{(i)}: \tilde{u}(x) \leq \frac{\lambda}{m}\right\} \\
\leq \operatorname{const}(P) \frac{\lambda^{2}}{m^{2}} \log ^{d-2} m
\end{gathered}
$$

Consequently

$$
\begin{aligned}
& \sum_{\lambda=0}^{\lambda_{0}} \lambda^{d-1} e^{-\lambda / 2} \operatorname{meas}\left\{(x, y) \in K_{3}^{(i)}: \tilde{u}(x) \leq \frac{\lambda}{m}\right\} \\
& \leq \operatorname{const}(P) \frac{1}{m^{2}} \log ^{d-2} m
\end{aligned}
$$

and

$$
E_{3} \leq \operatorname{const}(P) \log ^{d-2} m
$$

\section{Proof of Theorem 1}

Consider a convex body $K \in \mathscr{K}_{1}^{d}$. Define $N(\varepsilon)$ as the maximal number of pairwise disjoint caps of $K$, each of volume $\varepsilon$. (A cap of $K$ is the intersection of $K$ with a halfspace.) If $K$ is a polytope, then $N(\varepsilon) \leq$ vert $K$ and $N(\varepsilon)=$ vert $K$ for small enough $\varepsilon$. Conversely we have:

$$
\text { If } N(\varepsilon) \text { is bounded, then } K \text { is a polytope. }
$$

To prove this assume that $N(\varepsilon) \leq N_{0}, N\left(\varepsilon_{0}\right)=N_{0}$, and take pairwise disjoint caps $C_{1}, \ldots, C_{N_{0}}$, each of volume $\varepsilon_{0}$. Then $C_{i}=K \cap H_{i}$ with a halfspace $H_{i}$. Write $H_{i}^{\varepsilon}$ for the halfspace contained in $H_{i}$ such that $\operatorname{vol}\left(K \cap H_{i}^{\varepsilon}\right)=\varepsilon$ for $0 \leq \varepsilon \leq \varepsilon_{0}$. By changing each $H_{i}$ a little and decreasing $\varepsilon_{0}$ a little we may assume that $K \cap H_{i}^{0}$ is a single point $z_{i}$. We show now that $K=\operatorname{conv}\left\{z_{1}, \ldots, z_{N_{0}}\right\}$. Assume not, then there is a point $z_{0}$ on the boundary of $K$ with $z_{0} \notin \operatorname{conv}\left\{z_{1}, \ldots, z_{N_{0}}\right\}$. Then there is a halfspace $H_{0}$ with $z_{0} \in$ int $H_{0}$ and $z_{i} \notin H_{0}\left(i=1, \ldots, N_{0}\right)$. Then the cap $H_{0}^{\varepsilon} \cap K$ 
is disjoint from all the other caps $H_{i}^{\varepsilon} \cap K$ for sufficiently small $\varepsilon$, a contradiction proving (7.1).

Now we prove (1.2). Let first $K$ be a polytope. If it is not a simplex, it has at least $d+2$ vertices, each vertex belongs to at least $d$ edges, and, generally, each $k$-face belongs to at least $d-k$ faces of dimension $k+1$. Hence $T(K) \geq(d+2) d$ !, and Theorem 2 gives

$$
\lim \inf \frac{E(K, n)}{E(\Delta, n)}=\frac{T(K)}{T(\Delta)} \geq \frac{(d+2) d !}{(d+1) !}=1+\frac{1}{d+1}
$$

unless $K$ is a simplex. So assume $K$ is not a polytope. For $\varepsilon>0$ small, find $N(\varepsilon)$ and pairwise disjoint caps $C_{1}, \ldots, C_{N(\varepsilon)}$ of volume $\varepsilon$. Let $C_{i}=K \cap H_{i}$ and $C_{i}^{*}=K \cap H_{i}^{*}$ where the halfspace $H_{i}^{*}$ is contained in $H_{i}$ with its boundary hyperplane halving the width of $C_{i}$ in direction orthogonal to $H_{i}$. Clearly, for $\eta>0$ small enough

$$
\left\{x \in C_{i}^{*}: u_{C_{i}}(x) \leq \eta\right\}=\left\{x \in C_{i}^{*}: u_{K}(x) \leq \eta\right\} .
$$

The proof of Theorem 2 of [BáLa], applied to $C_{i}$ (cf. (2.2)), yields

$$
\operatorname{vol}\left\{x \in C_{i}^{*}: u_{C_{i}}(x) \leq \eta\right\} \geq \operatorname{const}(d) \eta \log ^{d-1} \frac{\varepsilon}{\eta} .
$$

Choosing $\varepsilon=\sqrt{\eta}$ we obtain

$$
\begin{aligned}
\operatorname{vol} K\left(u_{K} \leq \eta\right) & \geq \sum_{i=1}^{N(\sqrt{\eta})} \operatorname{vol}\left\{x \in C_{i}^{*}: u_{K}(x) \leq \eta\right\} \\
& =\sum_{i=1}^{N(\sqrt{\eta})} \operatorname{vol}\left\{x \in C_{i}^{*}: u_{C_{i}}(x) \leq \eta\right\} \\
& \geq \operatorname{const}(d) N(\sqrt{\eta}) \eta \log ^{d-1} \frac{1}{\eta}
\end{aligned}
$$

and consequently, by (2.1),

$$
E(K, n) \geq \operatorname{const}(d) N\left(\frac{1}{\sqrt{n}}\right) \frac{\log ^{d-1} n}{n} .
$$

Since $N\left(\frac{1}{\sqrt{n}}\right)$ is unbounded by $(7.1)$, this shows that

$$
\liminf \frac{E(K, n)}{E(\Delta, n)} \geq \liminf \operatorname{const}(d) N\left(\frac{1}{\sqrt{n}}\right)=\infty .
$$

\section{Proof of the lemmata}

Proof of Lemma 1. The set $K(v \geq \varepsilon)$ is convex as it is the intersection of closed halfspaces. By Lemma $F$ of [Bá] it does not contain any line segment on its boundary provided $\varepsilon>0$. Therefore the maximal $v$-value on aff $(a, b)$ is attained at a unique point $c^{*}$, and there is a hyperplane $H^{*}$ containing aff $(a, b)$ such that $K\left(v \geq v\left(c^{*}\right)\right) \cap H^{*}=$ $\left\{c^{*}\right\}$. From Lemma $G$ of [Bá] we know that if $C$ is a cap with $K(v \geq \varepsilon) \cap C=\{x\}$, 
a single point, then $C \subset M(x, 3 d)$ provided $\varepsilon$ is sufficiently small. Hence the cap $C^{*}$ cut off from $K$ by $H^{*}$ is contained in $M\left(c^{*}, 3 d\right)$, and consequently

$$
\frac{\left\|a-c^{*}\right\|}{\left\|b-c^{*}\right\|} \leq 3 d
$$

Now if $c^{*}$ is on the line segment connecting $c$ and $b$, clearly

$$
\frac{\|a-c\|}{\|b-c\|} \leq \frac{\left\|a-c^{*}\right\|}{\left\|b-c^{*}\right\|} \leq 3 d
$$

and we are done. So assume $c^{*}$ is on the line segment connecting $c$ and $a$. Since $u$ is maximal at $c, u(c) \geq u\left(c^{*}\right)$. Write $Q^{*}=K \cap H^{*}$. Let the width of $C^{*}$ be $h$ in the direction orthogonal to $H^{*}$. As $C^{*} \subset M\left(c^{*}, 3 d\right)$, the width of $M\left(c^{*}\right)$ in the same direction is at least $\frac{2}{3 d} h$. Considering (3.2), (3.3), and (3.4) we see that

$$
\begin{aligned}
u(c) & \leq 2 h u_{Q^{*}}(c), \\
u\left(c^{*}\right) & \geq \frac{1}{d} \frac{2}{3 d} h u_{Q^{*}}\left(c^{*}\right) .
\end{aligned}
$$

Let $L$ be the $(d-2)$-dimensional plane in $H^{*}$ through $b$ orthogonal to aff $(a, b)$, and let $\sigma$ be the maximal $(d-2)$-dimensional volume of a section of $Q^{*}$ with a plane that is parallel to $L$. Then

$$
u_{Q^{*}}(c) \leq 2\|b-c\| \sigma .
$$

On the other hand, $C^{*} \subset M\left(c^{*}, 3 d\right)$ implies $Q^{*} \subset M_{Q^{*}}\left(c^{*}, 3 d\right)$ and thus $\operatorname{vol}_{d-1} Q^{*} \leq$ $(3 d)^{d-1} \operatorname{vol}_{d-1} M_{Q^{*}}\left(c^{*}\right)$, i.e.

$$
u_{Q^{*}}\left(c^{*}\right) \geq \frac{1}{(3 d)^{d-1}} \operatorname{vol}_{d-1} Q^{*} .
$$

As $\operatorname{vol}_{d-1} Q^{*} \geq \frac{1}{d-1}\|a-b\| \sigma$

$$
u_{Q^{*}}\left(c^{*}\right) \geq \frac{1}{(d-1)(3 d)^{d-1}}\|a-b\| \sigma .
$$

Hence

$$
\begin{aligned}
1 & \leq \frac{u(c)}{u\left(c^{*}\right)} \leq 3 d^{2} \frac{u_{Q^{*}}(c)}{u_{Q^{*}}\left(c^{*}\right)} \leq 6 d^{2}(d-1)(3 d)^{d-1} \frac{\|b-c\|}{\|a-b\|} \\
& \leq(3 d)^{d+2} \frac{\|b-c\|}{\|a-b\|}
\end{aligned}
$$

and $\frac{\|a-b\|}{\|b-c\|} \leq(3 d)^{d+2}$ gives $\frac{\|a-c\|}{\|b-c\|} \leq(3 d)^{d+2}$.

Proof of Lemma 2. Set, as in the proof of Theorem 4, $K=P\left(\bar{\phi}_{i-1}\right)$ and $K\left(\tau_{i} \geq \phi_{i}\right)=$ $P\left(\bar{\phi}_{i-1}, \tau_{i} \geq \phi_{i}\right)$. We may assume $\tau_{0}(x) \leq \frac{\phi_{0}}{\theta+1}$ which implies that $K\left(\tau_{0} \geq \phi_{0}\right) \cap$ $M_{K}(x, \theta)$ is empty, proving the lemma when $i=0$.

For $i \geq 1$ we first consider the case $\theta=1$. Recall the definition of $Q$ in (1.8), set $q=$ cone $F_{1} \cap H_{0}\left(t_{0}\right)$ and define

$$
x^{*}=x+\left(1-\tau_{0}(x) t_{0}^{-1}\right) q .
$$


Assume now $i>1$. It is not difficult to see that for $0 \leq t \leq 2 \tau_{0}(x)$

$$
M_{K}(x) \cap H_{0}(t) \subseteq\left(-1+t t_{0}^{-1}\right) q+M_{Q\left(\phi_{1}, \ldots, \phi_{i-1}\right)}\left(x^{*}\right) .
$$

$\left(M_{K}(x) \cap H_{0}(t)\right.$ is empty if $t>2 \tau_{0}(x)$.) From

$$
K\left(\tau_{i} \geq \phi_{i}\right)=\operatorname{cone} Q\left(\phi_{1}, \ldots, \phi_{i-1}, \tau_{i-1}^{(Q)} \geq \phi_{i}\right) \cap H_{0}\left(0, \phi_{0}\right)
$$

(cf. (2.8)) it follows that for $0 \leq t \leq t_{0}$

$$
K\left(\tau_{i} \geq \phi_{i}\right) \cap H_{0}(t) \subseteq\left(-1+t t_{0}^{-1}\right) q+Q\left(\phi_{1}, \ldots, \phi_{i-1} ; \tau_{i-1}^{(Q)} \geq \phi_{i}\right) .
$$

(8.1), (8.2), and the induction hypothesis yield

$$
\begin{aligned}
\operatorname{vol}_{d-1} & {\left[K\left(\tau_{i} \geq \phi_{i}\right) \cap M_{K}(x) \cap H_{0}(t)\right] } \\
& =\operatorname{vol}_{d-1}\left[Q\left(\phi_{1}, \ldots, \phi_{i-1} ; \tau_{i-1}^{(Q)} \geq \phi_{i}\right) \cap M_{Q\left(\phi_{1}, \ldots, \phi_{i-1}\right)}\left(x^{*}\right)\right] \\
& \leq \operatorname{const}(Q) \tau_{i-1}^{(Q)}\left(x^{*}\right) \operatorname{vol}_{d-1} M_{Q\left(\phi_{1}, \ldots, \phi_{i-1}\right)}\left(x^{*}\right) \\
& =\operatorname{const}(Q) \tau_{i}(x) \operatorname{vol}_{d-1} M_{Q\left(\tau_{0}(x)\right)\left(\phi_{1}, \ldots, \phi_{i-1}\right)}(x)
\end{aligned}
$$

since $\tau_{i-1}^{(Q)}\left(x^{*}\right)=\tau_{i}(x)$ as $i>1$ (cf. $\left.(2.5)\right)$ and $M_{Q\left(\phi_{1}, \ldots, \phi_{i-1}\right)}\left(x^{*}\right)$ is congruent to $M_{Q\left(\tau_{0}(x)\right)\left(\phi_{1}, \ldots, \phi_{i-1}\right)}(x)$. Then

$$
\begin{aligned}
& \operatorname{vol}\left[K\left(\tau_{i} \geq \phi_{i}\right) \cap M_{K}(x)\right] \\
& \quad=\int_{0}^{2 \tau_{0}(x)} \operatorname{vol}_{d-1}\left[K\left(\tau_{i} \geq \phi_{i}\right) \cap M_{K}(x) \cap H_{0}(t)\right] d t \\
& \quad \leq 2 \tau_{0}(x) \operatorname{const}(Q) \tau_{i}(x) \operatorname{vol}_{d-1} M_{Q\left(\tau_{0}(x)\right)\left(\phi_{1}, \ldots, \phi_{i-1}\right)}(x) \\
& \quad \leq \operatorname{const}(P) \tau_{i}(x) \operatorname{vol} M_{K}(x),
\end{aligned}
$$

where the last step follows from (3.4).

Special care is needed when $i=1$. Then the hyperplane $H\left(F_{1}\right)$ supports $K$ and so $M_{K}(x)$ lies between the hyperplanes $H\left(F_{1}\right)$ and $2 x-H\left(F_{1}\right)$ which is the reflection of $H\left(F_{1}\right)$ through $x$. The slab between these hyperplanes intersects $Q$ in $Q\left(\tau_{0}^{(Q)} \leq 2 \tau_{0}(x) \tau_{1}(x) t_{0}^{-1}\right)$. So we have instead of (8.1)

$$
M_{K}(x) \cap H_{0}(t) \subseteq\left(-1+t t_{0}^{-1}\right) q+Q\left(\tau_{0}^{(Q)} \leq 2 \tau_{0}(x) \tau_{1}(x) t_{0}^{-1}\right) .
$$

On the other hand, using (2.4) we get

$$
K\left(\tau_{1} \geq \phi_{1}\right) \cap H_{0}(t) \subseteq\left(-1+t t_{0}^{-1}\right) q+Q\left(\tau_{0}^{(Q)} \geq t \phi_{1} t_{0}^{-1}\right)
$$

Hence $K\left(\tau_{1} \geq \phi_{1}\right) \cap M_{K}(x) \cap H_{0}(t)$ is empty unless $t \phi_{1} t_{0}^{-1} \leq 2 \tau_{0}(x) \tau_{1}(x) t_{0}^{-1}$. Thus 


$$
\begin{aligned}
\operatorname{vol}\left[K\left(\tau_{1} \geq \phi_{1}\right) \cap M_{K}(x)\right] \\
=\int_{0}^{2 \tau_{0}(x)} \operatorname{vol}_{d-1}\left[K\left(\tau_{1} \geq \phi_{1}\right) \cap M_{K}(x) \cap H_{0}(t)\right] d t \\
\quad \leq \int_{0}^{2 \tau_{0}(x) \tau_{1}(x) \phi_{1}^{-1}} \operatorname{vol}_{d-1}\left[M_{K}(x) \cap H_{0}(t)\right] d t \\
\leq 2 \tau_{0}(x) \tau_{1}(x) \phi_{1}^{-1} \operatorname{vol}_{d-1}\left[M_{K}(x) \cap H_{0}\left(\tau_{0}(x)\right)\right] \\
\leq d \phi_{1}^{-1} \tau_{1}(x) \operatorname{vol} M_{K}(x)
\end{aligned}
$$

If $\theta>1, x+\theta(K-x) \supset K \supset K\left(\tau_{i} \geq \phi_{i}\right)$ implies

$$
\begin{aligned}
& K\left(\tau_{i} \geq \phi_{i}\right) \cap M_{K}(x, \theta) \\
& =K\left(\tau_{i} \geq \phi_{i}\right) \cap\{x+\theta[(K-x) \cap(x-K)]\} \\
& =K\left(\tau_{i} \geq \phi_{i}\right) \cap[x+\theta(K-x)] \cap[x+\theta(x-K)] \\
& =K\left(\tau_{i} \geq \phi_{i}\right) \cap[x+(K-x)] \cap[x+\theta(x-K)],
\end{aligned}
$$

and as $K=$ cone $Q\left(\phi_{1}, \ldots, \phi_{i-1}\right) \cap H_{0}\left(0, \phi_{0}\right)$, it follows from $\tau_{0}(x) \leq \frac{\phi_{0}}{\theta+1}$ that

$$
\begin{aligned}
& {[x+(K-x)] \cap[x+\theta(x-K)]} \\
& =K \cap[(\theta+1) x-\theta K] \\
& =\frac{\theta+1}{2} x+\left[\left(K-\frac{\theta+1}{2} x\right) \cap\left(\frac{\theta+1}{2} x-K\right)\right] \\
& =M_{K}\left(\frac{\theta+1}{2} x, 1\right) .
\end{aligned}
$$

Consequently

$$
K\left(\tau_{i} \geq \phi_{i}\right) \cap M_{K}(x, \theta)=K\left(\tau_{i} \geq \phi_{i}\right) \cap M_{K}\left(\frac{\theta+1}{2} x, 1\right) .
$$

On the other hand, $\tau_{i}\left(\frac{\theta+1}{2} x\right)=\tau_{i}(x)$ and

$$
\begin{aligned}
M_{K}\left(\frac{\theta+1}{2} x, 1\right) & =[x+(K-x)] \cap[x+\theta(x-K)] \\
& \subset[x+\theta(K-x)] \cap[x+\theta(x-K)] \\
& =M_{K}(x, \theta) .
\end{aligned}
$$

Thus we have

$$
\begin{aligned}
\operatorname{vol}[ & \left.K\left(\tau_{i} \geq \phi_{i}\right) \cap M_{K}(x, \theta)\right] \\
& =\operatorname{vol}\left[K\left(\tau_{i} \geq \phi_{i}\right) \cap M_{K}\left(\frac{\theta+1}{2} x, 1\right)\right] \\
& \leq \operatorname{const}(P) \tau_{i}\left(\frac{\theta+1}{2} x\right) \operatorname{vol} M_{K}\left(\frac{\theta+1}{2} x, 1\right) \\
& \leq \operatorname{const}(P) \tau_{i}(x) \operatorname{vol} M_{K}(x, \theta) .
\end{aligned}
$$


Proof of Lemma 3. We are going to use Theorem 6 of [BáLa] and Theorems 7 and 8 of [Bá]. They - or rather their proofs - say the following:

For a convex body $K \subset \mathscr{K}_{1}^{d}$ and $\varepsilon \leq \varepsilon_{0}(d)$ assume that $z_{1}, \ldots, z_{N}$ is a system of points maximal with respect to the following two properties: $u\left(z_{j}\right)=\varepsilon$ for every $j=1, \ldots, N$ and $M\left(z_{j}, \frac{1}{2}\right) \cap M\left(z_{k}, \frac{1}{2}\right)=\emptyset$ for every $j, k=1, \ldots, N, j \neq k$. According to Macbeath, the set $K(u \geq \varepsilon)$ is convex (recall Section 2) and does not contain any line segment on its boundary (recall Section 3), so for every $z_{j}$ there is a halfspace $H_{j}^{+}$with $K(u \geq \varepsilon) \cap H_{j}^{+}=\left\{z_{j}\right\}$. Now, by Theorem 6 of [BáLa]

$$
\bigcup_{j=1}^{N}\left[M\left(z_{j}, \frac{1}{2}\right) \cap H_{j}^{+}\right] \subset K(u \leq \varepsilon) \subset \bigcup_{j=1}^{N} M\left(z_{j}, 5\right),
$$

and by Theorems 7 and 8 of [Bá]

$$
\{(x, y) \in K \times K: u(x, y) \leq \varepsilon\} \subset \bigcup_{j=1}^{N} M\left(z_{j}, 15 d\right) \times M\left(z_{j}, 15 d\right) .
$$

Again set $K=P\left(\bar{\phi}_{i-1}\right)$ and $K\left(\tau_{i} \geq \phi_{i}\right)=P\left(\bar{\phi}_{i-1}, \tau_{i} \geq \phi_{i}\right)$. As $K$ is a polytope, by Theorem 6 , vol $K(u \leq \varepsilon) \leq \operatorname{const}(P) \varepsilon \log ^{d-1} \frac{1}{\varepsilon}$. On the other hand, $\operatorname{vol}\left[M\left(z_{j}, \frac{1}{2}\right) \cap\right.$ $\left.H_{j}^{+}\right]=2^{-(d+1)} \varepsilon$. Hence

$$
N \leq \operatorname{const}(P) \log ^{d-1} \frac{1}{\varepsilon} .
$$

Claim. If $z \notin S(T, 2 \eta)$ and $H^{+}$is any halfspace containing $z$ in its bounding hyperplane, then

$$
\operatorname{vol}\left[M\left(z, \frac{1}{2}\right) \cap H^{+} \backslash S(T, \eta)\right] \geq \frac{1}{d ! 2^{d}} \operatorname{vol} M\left(z, \frac{1}{2}\right) .
$$

Proof. By induction on $d$. The case $d=1$ is trivial. Since

$$
M\left(z, \frac{1}{2}\right) \subset H_{0}\left(\frac{1}{2} \tau_{0}(z), \frac{3}{2} \tau_{0}(z)\right)
$$

and the last set is disjoint from $S(T, \eta)$ whenever $\eta<\frac{1}{2} \tau_{0}(z)$, only the case $\tau_{0}(z) \leq 2 \eta$ has to be considered.

As $z \in H_{0}(0,2 \eta)$ and $z \notin S(T, 2 \eta)=$ cone $S_{Q}\left(T_{Q}, 2 \eta\right) \cap H_{0}(0,2 \eta)$ (cf. (1.11)), clearly $z_{Q} \notin S_{Q}\left(T_{Q}, 2 \eta\right)$ (cf. (2.3)). Then, by the induction hypothesis, for any halfspace $H_{Q}^{+}$in $H_{0}\left(t_{0}\right)$ containing $z_{Q}$ on its boundary

$$
\operatorname{vol}_{d-1}\left[M_{Q}\left(z_{Q}, \frac{1}{2}\right) \cap H_{Q}^{+} \backslash S_{Q}\left(T_{Q}, \eta\right)\right] \geq \frac{1}{(d-1) ! 2^{d-1}} \operatorname{vol}_{d-1} M_{Q}\left(z_{Q}, \frac{1}{2}\right) .
$$

Choosing $H_{Q}^{+}:=$cone $\left[H^{+} \cap H_{0}\left(\tau_{0}(z)\right)\right] \cap H_{0}\left(t_{0}\right)$ and replacing $H_{0}\left(t_{0}\right)$ by $H_{0}\left(\tau_{0}(z)\right)$ we obtain

$$
\begin{array}{r}
\operatorname{vol}_{d-1}\left[M\left(z, \frac{1}{2}\right) \cap H_{0}\left(\tau_{0}(z)\right) \cap H^{+} \backslash \text { cone } S_{Q}\left(T_{Q}, \eta\right)\right] \\
\geq \frac{1}{(d-1) ! 2^{d-1}} \operatorname{vol}_{d-1}\left[M\left(z, \frac{1}{2}\right) \cap H_{0}\left(\tau_{0}(z)\right)\right] .
\end{array}
$$


(The set $H^{+} \cap H_{0}\left(\tau_{0}(z)\right)$ may, exceptionally, coincide with the whole $H_{0}\left(\tau_{0}(z)\right)$ ). In this case one has to perturb $H_{0}$.) The point $\frac{1}{2} z$ has distance $\frac{1}{2} \tau_{0}(z)$ from the $(d-1)$ dimensional set $M\left(z, \frac{1}{2}\right) \cap H_{0}\left(\tau_{0}(z)\right) \cap H^{+} \backslash$ cone $S_{Q}\left(T_{Q}, \eta\right)$. Both the point and the set lie in $M\left(z, \frac{1}{2}\right) \cap H^{+} \backslash S(T, \eta)$. Thus

$$
\begin{aligned}
\operatorname{vol}[ & \left.M\left(z, \frac{1}{2}\right) \cap H^{+} \backslash S(T, \eta)\right] \\
& \geq \frac{1}{d} \frac{\tau_{0}(z)}{2} \operatorname{vol}_{d-1}\left[M\left(z, \frac{1}{2}\right) \cap H_{0}\left(\tau_{0}(z)\right) \cap H^{+} \backslash S(T, \eta)\right] \\
& \geq \frac{1}{d} \frac{\tau_{0}(z)}{2} \frac{1}{(d-1) ! 2^{d-1}} \operatorname{vol}\left[M\left(z, \frac{1}{2}\right) \cap H_{0}\left(\tau_{0}(z)\right)\right] \\
& \geq \frac{1}{d ! 2^{d}} \operatorname{vol} M\left(z, \frac{1}{2}\right)
\end{aligned}
$$

where the last step follows from (3.3).

The Claim shows that for $z_{j} \notin S(T, 2 \eta)$

$$
\operatorname{vol}\left[M\left(z, \frac{1}{2}\right) \cap H_{j}^{+} \backslash S(T, \eta)\right] \geq \frac{1}{d ! 4^{d}} \varepsilon .
$$

On the other hand, by $(4.4)$

$$
\operatorname{vol}\left[P(u \leq \varepsilon) \backslash \bigcup_{T} S(T, 2 \eta)\right] \leq \operatorname{const}(P) \varepsilon \log ^{d-2} \frac{1}{\varepsilon} \log \log \frac{1}{\varepsilon}
$$

if we choose $\eta=\left(\log \frac{1}{\varepsilon}\right)^{-1}$. Then (8.3) shows that the number of points $z_{j}$ outside $\cup_{T} S(T, 2 \eta)$ is at most

$$
\operatorname{const}(P) \log ^{d-2} \frac{1}{\varepsilon} \log \log \frac{1}{\varepsilon} .
$$

Further, (8.4) implies

$$
\begin{aligned}
& \left\{(x, y) \in P\left(\bar{\varphi}_{i}\right) \times K\left(\tau_{i} \geq \phi_{i}\right): u_{K}(x, y) \leq \varepsilon\right\} \\
& \subseteq \bigcup_{j=1}^{N}\left[M\left(z_{j}, 15 d\right) \cap P\left(\bar{\varphi}_{i}\right)\right] \times\left[M\left(z_{j}, 15 d\right) \cap K\left(\tau_{i} \geq \phi_{i}\right)\right] .
\end{aligned}
$$

Consider now a point $z_{j} \in S(T, 2 \eta)$ for some tower $T$. It follows from Lemma 2 that if the tower $T$ does not start with the chain of faces $F_{0} \subset F_{1} \subset \ldots \subset F_{i}$, then

$$
\operatorname{vol}\left[M\left(z_{j}, 15 d\right) \cap P\left(\bar{\varphi}_{i}\right)\right] \leq \operatorname{const}(P) \varepsilon\left(\log \frac{1}{\varepsilon}\right)^{-1} .
$$

When $T$ starts with this chain of faces, then, again by Lemma 2 ,

$$
\operatorname{vol}\left[M\left(z_{j}, 15 d\right) \cap K\left(\tau_{i} \geq \phi_{i}\right)\right] \leq \operatorname{const}(P) \varepsilon\left(\log \frac{1}{\varepsilon}\right)^{-1} .
$$

Taking the measure of the sets in (8.7) we get 


$$
\begin{aligned}
& \operatorname{meas}\left\{(x, y) \in P\left(\ddot{\varphi}_{i}\right) \times K\left(\tau_{i} \geq \phi_{i}\right): u_{K}(x, y) \leq \varepsilon\right\} \\
& \quad \leq \sum_{j=1}^{N} \operatorname{vol}\left[M\left(z_{j}, 15 d\right) \cap P\left(\vec{\varphi}_{i}\right)\right] \operatorname{vol}\left[M\left(z_{j}, 15 d\right) \cap K\left(\tau_{i} \geq \phi_{i}\right)\right] .
\end{aligned}
$$

By (8.6) there are at most const $(P) \log ^{d-2} \frac{1}{\varepsilon} \log \log \frac{1}{\varepsilon}$ terms with $z_{j} \notin \cup_{T} S(T, 2 \eta)$, and as both factors in each term are less than const $(d) \varepsilon$, the sum of these terms is at most

$$
\text { const }(P) \varepsilon^{2} \log ^{d-2} \frac{1}{\varepsilon} \log \log \frac{1}{\varepsilon} \text {. }
$$

By (8.8) the terms with $z_{j} \in S(T, 2 \eta)$ are less than $\operatorname{const}(P) \varepsilon\left(\log \frac{1}{\varepsilon}\right)^{-1}$ times const $(d) \varepsilon$ if $T$ does not start with $F_{0} \subset F_{1} \subset \ldots \subset F_{i}$, and by (8.9) less than const $(d) \varepsilon$ times const $(P) \varepsilon\left(\log \frac{1}{\varepsilon}\right)^{-1}$ if $T$ starts with $F_{0} \subset F_{1} \subset \ldots \subset F_{i}$. As by (8.5) there are at most const $(P) \log ^{d-1} \frac{1}{\varepsilon}$ terms, the sum of terms with $z_{j} \in \cup_{T} S(T, 2 \eta)$ is at most

$$
\operatorname{const}(P) \varepsilon^{2} \log ^{d-2} \frac{1}{\varepsilon}
$$

Therefore

$$
\begin{gathered}
\operatorname{meas}\left\{(x, y) \in P\left(\bar{\varphi}_{i}\right) \times K\left(\tau_{i} \geq \phi_{i}\right): u_{K}(x, y) \leq \varepsilon\right\} \\
\leq \operatorname{const}(P) \varepsilon^{2} \log ^{d-2} \frac{1}{\varepsilon} \log \log \frac{1}{\varepsilon} .
\end{gathered}
$$

\section{References}

[Af1] Affentranger, F.: The expected volume of a random polytope in a ball. J. Microscopy 151, 277-287 (1988)

[Af2] Affentranger, F.: Aproximación aleatoria de cuerpos.convexos. Publ. Mat., Barc. 36, 85-109 (1992)

[AW] Affentranger, F., Wieacker, J. A.: On the convex hull of uniform random points in a simple d-polytope. Discrete Comput. Geom. 6, 291-305 (1991)

[Bá] Bárány, l.: Intrinsic volumes and $f$-vectors of random polytopes. Math. Ann. 285, 671-699 (1989)

[BB] Bárany, I., Buchta, C.: On the convex hull of uniform random points in an arbitrary $d$-polytope. Anz. Österr. Akad. Wiss., Math.-Naturwiss. K1. 127, 25-27 (1990)

[BáLa] Bárány, I., Larman, D. G.: Convex bodies, economic cap coverings, random polytopes. Mathematika 35, 274-291 (1988)

[BaLe] Bayer, M. M., Lee, C. W.: Convex polytopes. In: Gruber, P. M., Wills, J. M. (eds.) Handbook of convex geometry. Amsterdam: Elsevier 1993

[BKST] Bentley, J. L., Kung, H. T., Schkolnick, M., Thompson, C. D.: On the average number of maxima in a set of vectors and applications. J. Assoc. Comput. Mach. 25, 536-543 (1978)

[BI1] Blaschke, W.: Über affine Geometrie XI: Lösung des "Vierpunktproblems" von Sylvester aus der Theorie der geometrischen Wahrscheinichkeiten. Ber. Verh. Sächs. Ges. Wiss. Leipzig, Math.Phys. Kl. 69, 436-453 (1917)

[B12] Blaschke, W.: Vorlesungen ubet Differentialgeometrie, vol. II. Affine Differentialgeometrie. Berlin: Springer 1923

[Bu1] Buchta, C.: Über die konvexe Hülle von Zufallspunkten in Eibereichen. Elem. Math. 38, 153-156 (1983)

[Bu2] Buchta, C.: Zufallspolygone in konvexen Vielecken. J. Reine Angew. Math. 347, 212-220 (1984)

[Bu3] Buchta, C.: Zufallige Polyeder - Eine Übersicht. In: Hlawka, E. (ed.) Zahlentheoretische Analysis (Lect. Notes Math., vol. 1114, pp. 1-13) Berlin Heidelberg New York Tokyo: Springer 1985

[Bu4] Buchta, C.: A note on the volume of a random polytope in a tetrahedron. Illinois J. Math. 30, $653-659(1986)$ 
[BR] Buchta, C., Reitzner, M.: What is the expected volume of a tetrahedron whose vertices are chosen at random from a given tetrahedron? Anz. Österr. Akad. Wiss., Math.-Naturwiss. KI. 129, 63-68 (1992)

[CFG] Croft, H. T., Falconer, K. J., Guy, R. K.: Unsolved problems in geometry. New York Berlin Heidelberg: Springer 1991

[DL] Dalla, L., Larman, D. G.: Volumes of a random polytope in a convex set. In: Gritzmann, P., Sturmfels, B. (eds.) Applied geometry and discrete mathematics: The Victor Klee Festschrift (DIMACS Series in Discrete Mathematics and Theoretical Computer Science, vol. 4, pp. 175180) Providence, RI: Am. Math. Soc. 1991

[De] Devroye, L.: A note on finding convex hulls via maximal vectors. Inf. Process. Lett. 11, 53-56 (1980)

[Dw] Dwyer, R. A.: On the convex hull of random points in a polytope. J. Appl. Probab. 25, 688-699 (1988)

[DK] Dwyer, R. A., Kannan, R.: Convex hull of randomly chosen points from a polytope. Math. Res. 38, 16-24 (1987)

[Ef] Efron, B.: The convex hull of a random set of points. Biometrika 52, 331-343 (1965)

[ELR] Ewald, G., Larman, D. G., Rogers, C. A.: The directions of the line segments and of the $r$ dimensional balls on the boundary of a convex body in Euclidean space. Mathematika 17, 1-20 (1970)

[Fe] Feller, W.: An introduction to probability theory and its applications, vol. II. New York London Sydney: Wiley 1966

[Gi] Giannopoulos, A. A.: On the mean value of the area of a random polygon in a plane convex body. Mathematika 39, 279-290 (1992)

[Gr1] Groemer, H.: On the mean value of the volume of a random polytope in a convex set. Arch. Math. 25, 86-90 (1974)

[Gr2] Groemer, H.: Math. Reviews 84g: 60019 (1984)

[Ma] Macbeath, A. M.: A theorem on non-homogeneous lattices. Ann. Math., II. Ser. 56, 269-293 (1952)

[Pf] Pfiefer, R. E.: The historical development of J. J. Sylvester's four point problem. Math. Mag. 62, 309-317 (1989)

[Re] Reed, W. J.: Random points in a simplex. Pacific J. Math. 54, 183-198 (1974)

[RS] Rényi, A., Sulanke, R.: Über die konvexe Hülle von $n$ zufällig gewählten Punkten. Z. Wahrscheinlichkeitsth. Verw. Geb. 2, 75-84 (1963)

[Schn] Schneider, R.: Random approximation of convex sets. J. Microscopy 151, 211-227 (1988)

[Schü] Schütt, C.: The convex floating body and polyhedral approximation. Israel J. Math. 73, 65-77 (1991)

[We] Van Wel, B. F.: The convex hull of a uniform sample from the interior of a simple $d$-polytope. J. Appl. Probab. 26, 259-273 (1989)

[WW] Weil, W., Wieacker, J. A.: Stochastic geometry. In: Gruber, P. M., Wills, J. M. (eds.) Handbook of convex geometry. Amsterdam: Elsevier 1993

[Wi] Wieacker, J. A.: Einige Probleme der polyedrischen Approximation. Diplomarbeit, AlbertLudwigs-Universität, Freiburg im Breisgau 1978

This article was processed by the authors using the Springer-Verlag TEX PJourlg macro package 1991. 